\title{
On the IAA version of the Doi-Edwards model versus the K-BKZ rheological model for polymer fluids: a global existence result for shear flows with small initial data. *
}

\author{
Ionel Sorin Ciuperca ${ }^{1}$, Arnaud Heibig ${ }^{2}$ and Liviu Iulian Palade ${ }^{2}$
}

July 17,2018

Université de Lyon, CNRS, Institut Camille Jordan UMR 5208

${ }^{1}$ Université Lyon 1, Bât Braconnier, 43 Boulevard du 11 Novembre 1918, F-69622,

Villeurbanne, France.

${ }^{2}$ INSA-Lyon, Pôle de Mathématiques, Bât. Leonard de Vinci No. 401, 21 Avenue Jean Capelle, F-69621, Villeurbanne, France.

\begin{abstract}
This paper establishes the existence of smooth solutions for the Doi-Edwards rheological model of viscoelastic polymer fluids in shear flows. The problem turns out to be formally equivalent to a K-BKZ equation but with constitutive functions spanning beyond the usual mathematical framework. We prove, for small enough initial data, that the solution remains in the domain of hyperbolicity of the equation for all $t \geq 0$.
\end{abstract}

Keywords: Doi-Edwards polymer model; K-BKZ viscoelastic fluid; shear flows; convolution operator; evolutionary integro-differential equation.

\section{Introduction.}

Modeling industrial flows of non-Newtonian and viscoelastic fluids using molecular models and theories is nowadays of utter importance, see e.g. [1], 22, [4], [5], [7], 11], 14], 15], [16], [17, [21], [25], [27], [28], [31], 33]. However, this activity success hinges on users ability to cope with significant mathematical difficulties. This paper presents existence results for the shear flow of a Doi-Edwards / K-BKZ fluid, and before getting to the matter, for sake of clarity, we quickly introspect the key physical considerations laying at the model foundation.

In the realm of unfilled polymeric liquids, one distinguishes dilute systems from concentrated and melt ones and notices a physico-mathematical divide: constitutive laws and related mathematical technicalities are to a good extent different. When polymer concentration increases beyond a critical value, molecules overlap and excluded volume interactions, hydrodynamic interactions and entanglement interactions all strongly affect the molecular motion making calculations significantly complicated. At the present, there are two theories - which are reasonably supported by experiments - that account for the molecular dynamics, called the density

*Dedicated to Professor Denis Serre, Ecole Normale Supérieure de Lyon, France, on the occasion of his 60th birthday anniversary.

${ }^{\dagger}$ Corresponding author. E-mail: ciuperca@math.univ-lyon.fr, arnaud.heibig@insa-lyon.fr, liviuiulian.palade@insa-lyon.fr; Fax: +33 472438529 
(concentration) fluctuation theory and the kinetical theory. Although they describe different aspects of the dynamics, they are nonetheless seen as related to each other; however, the interrelation is far from being conspicuous and remains a matter of debate. The kinetical theory of Bird, Curtiss, Armstrong and Hassager [6] on one hand, and that of Doi and Edwards [20] (DE for the short) based on de Gennes' reptation diffusion [18] on the other, are considered of paramount importance. Here we shall talk about the later.

In the DE model, a polymer chain - which consists of articulated segments - is seen as confined within a tube made-up of the surrounding chains and is free to wriggle out of it under imposed strain in a snake-like diffusion called reptation. As Doi and Edwards put it, this is not to say the tube vanished, but the protruding part moves into a newly formed tube segment while the portion that has been vacated on this occasion ceases to exist. Put it otherwise, the chain uses the degrees of freedom of its extremities to gradually change its shape by reptating. Under the influence of an external field, both the macromolecule and the tube are deformed in a cooperative manner. The chain configuration is actually determined by the new tubelike surrounding it just moved in, and is a many body problem of great complexity. Doi and Edwards first assumed that each chain segment deforms independently, hypothesis known as the Independent Alignment Approximation (IAA). The chain segment motion is seen as a concatenation of three processes which chronologically are: a) an instantaneous deformation, followed by b) a quick retraction to its original length, and eventually c) undergoes a slow reptation motion out of the tube it initially occupied. Processes a) and b) affect the chain orientation without stretching it, while a relaxation occurs at stage c). A more realistic view (albeit still an idealization of the real diffusion) is to consider d) that the deformation (i.e. the flow) causes an extension of the segments at a rate proportional to the macroscopic velocity gradient. In this case one needs to account for a retraction-extension mechanism by which the chain keeps its curvilinear length constant. When this is done, one gets what is commonly termed by physicists "the full nonlinear DE model", in the sense that a more complicated diffusion equation results in a more complex stress tensor expression, hence the nonlinear model.

Let $s \in(0, L)$ be the curvilinear coordinate along the chain, and $u=\left(u_{1}, u_{2}, u_{3}\right)$ a unitary vector belonging to the unit sphere $S_{2}$ that gives, for any $s$, the orientation of the chain segment.

At the heartcore of any kinetical model one finds a configurational probability diffusion equation the solution of which - here for convenience denoted $F$ - is needed to obtain the stress tensor (or put it differently, to get the corresponding constitutive equation, CE). The solution $F$ is a probability density with respect to the variable $u$, and solves ([13], [20], [35], [34]):

$$
\begin{aligned}
& \frac{\partial F}{\partial t}+\left(V \cdot \nabla_{X}\right) F=D \frac{\partial^{2} F}{\partial s^{2}}-\frac{\partial}{\partial u} \cdot[\mathscr{M}(t, u) F]+\epsilon F \kappa: u u-\epsilon \frac{\partial}{\partial s}[F \kappa: \lambda(F)], \\
& \text { for }(X, t, u, s) \in \widetilde{\Omega} \times(0,+\infty) \times S_{2} \times(0, L) \\
& F(s=0)=F(s=L)=\frac{1}{4 \pi} ; F(t=0)=F_{0}(X, s, u) \text { a given function, }
\end{aligned}
$$

where $(X, t, u, s) \in \widetilde{\Omega} \times(0,+\infty) \times S_{2} \times(0, L)$, and

$$
\lambda(F)(X, t, s)=\int_{0}^{s}\left[\int_{S_{2}} F\left(X, t, u, s^{\prime}\right) u u \mathrm{~d} \sigma\right] \mathrm{d} s^{\prime}
$$

and

$$
\mathscr{M}(t, u)=\kappa \cdot u-(\kappa: u u) u .
$$

In the above, $D>0$ the diffusion coefficient, $X=\left(x_{1}, x_{2}, x_{3}\right) \in \widetilde{\Omega} \subset \mathbb{R}^{3}$ is the macroscopic Eulerian variable, $t$ the time. $F=F(X, t, u, s)$ is the configurational probability, $\kappa=\nabla_{X} V$ the velocity gradient, and $\epsilon \geq 0$ a model parameter. Einstein's usual summation convention over 
repeated indices applies wherever needed; classical tensor calculus notations are also used, e.g. $u u$ stands for $u \otimes u$, etc. Note the presence of velocity $V$ in equation (1.1). If one sets $\epsilon=0$, the simplified diffusion equation is proper to the IAA version of the DE model, where chain segment extension-retraction mechanism is neglected. If $\epsilon>0$, then all mechanisms from a) to d) are taken into consideration and one deals with a nonlinear diffusion equation. A more detailed discussion on the physical meaning of the four terms in the r.h.s. of 1.2 is given e.g. in [13], 20], 35], 34].

The stress tensor $\tilde{\Sigma}$ reads [13], [35], [34]:

$$
\begin{aligned}
& \tilde{\Sigma}=\alpha(H+\epsilon \widetilde{H}) \\
& H(X, t)=\frac{1}{L} \int_{S_{2}} \int_{0}^{L}(u u-\delta) F(X, t, u, s) \mathrm{d} s \mathrm{~d} \sigma \\
& \widetilde{H}(X, t)=\frac{1}{L} \int_{S_{2}} \int_{0}^{L} u u F(t, u, s, X) \ln [4 \pi F(X, t, u, s)] \mathrm{d} s \mathrm{~d} \sigma
\end{aligned}
$$

where $\alpha>0$ a physical constant; observe the stress tensor $\tilde{\Sigma}$ is made up of two parts $H$ and $\widetilde{H}$ given in 1.5 and $(1.6), \delta$ being the unit tensor.

The fluid flow is governed by the classical momentum balance equation:

$$
\begin{aligned}
& \frac{\partial V}{\partial t}+\left(V \cdot \nabla_{X}\right) V=-\nabla p+\nabla_{X} \cdot \tilde{\Sigma}+\tilde{f}, \text { in } \widetilde{\Omega} \\
& \nabla_{X} \cdot V=0, \text { in } \widetilde{\Omega}
\end{aligned}
$$

with $p$ denoting the pressure. For the system of equations (1.1)- (1.7), energy and entropy estimates can be obtained by usual means. However, estimates on higher order derivatives are necessary in order to prove the existence of a solution to the aforementioned system.

In [13] we proved the existence and uniqueness of solutions to the diffusion equation (1.1) assuming $V(t)$ is given and independent of $X$, using the Schauder fixed point theorem and the Galerkin's approximation method (for the existence and uniqueness of solutions of the corresponding stationary equation see [12]).

Compared to this manuscript framework, previously published papers on other polymer molecular models - e.g. [3], [26], [30], 31], 33] or [42] - deal with a different type of system of equations in that one notices the presence of Newtonian fluid related term, i.e. the Laplacian $\Delta_{X} V$, in the corresponding momentum balance equations. There, this term originates from physical considerations regarding the choice of a suitable constitutive law for the stress tensor $\tilde{\Sigma}$. Specifically, if one deals with a dilute polymeric fluid, then it is customary to assume that the total Cauchy extra-stress tensor is given by the sum between the solvent contribution which generally behaves like an ideal Newtonian fluid - hence the $\Delta_{X} V$ term - and a purely non-Newtonian or viscoelastic contribution. This assumption is in excellent agreement with experimental observations on dilute polymer liquids. However, in this work case, the fluid under scrutiny is solvent-free. Consequently there is no Newtonian contribution to the stress tensor and we must deal with the additional difficulty that springs off from the absence of the otherwise convenient $\Delta_{X} V$ term.

In view of this difficulty, we choose here to solve the important problem of existence of a solution to (1.1)-1.7) in the case of a simple shear flow for which $V(X, t)=\left(v\left(x_{2}, t\right), 0,0\right)$, $\left.F=F\left(x_{2}, t, u, s\right), p=p\left(x_{2}, t\right), \tilde{f}=\left(f\left(x_{2}, t\right), 0,0\right)\right)$, and taking $\epsilon=0$ which corresponds to the IAA version of the model. For simplicity, from here on we denote $x_{2}$ by $x$ : e.g. $v\left(x_{2}, t\right)$ reads $v(x, t)$. 
The governing equations for the shear flow are given below:

$$
\begin{gathered}
\frac{\partial v}{\partial t}=\frac{\partial \tilde{\Sigma}_{12}}{\partial x}+f \\
\frac{\partial p}{\partial x}=\frac{\partial \tilde{\Sigma}_{22}}{\partial x} \\
\frac{\partial \tilde{\Sigma}_{32}}{\partial x}=0
\end{gathered}
$$

where:

$$
\begin{gathered}
\tilde{\Sigma}_{j 2}=\frac{1}{L} \int_{0}^{L} \int_{S_{2}} u_{2} u_{j} F \mathrm{~d} u \mathrm{~d} s-\frac{4 \pi}{3} \delta_{j 2}, j=1,2,3 \\
\frac{\partial F}{\partial t}=\frac{\partial^{2} F}{\partial s^{2}}-\frac{\partial v}{\partial x} \frac{\partial}{\partial u} \cdot\left(\mathcal{H}_{0}(u) F\right)
\end{gathered}
$$

In the above, $\mathcal{H}_{0}(u)=M_{0} \cdot u-\left(M_{0}: u u\right) u$, with $\left(M_{0}\right)_{i j}=\delta_{i 1} \delta_{2 j}$.

To the system of equations (1.8)- 1.12 we assign the following boundary and initial conditions:

$$
\left\{\begin{array}{l}
v=0, \text { for } x \in \partial \Omega \\
v=v_{0}, \text { for } t=0 \\
F=\frac{1}{4 \pi}, \text { for } s=0 \text { or } s=L \\
F=F_{0}, \text { for } t=0
\end{array}\right.
$$

where $\Omega \subset \mathbb{R}$ is the range for $x$, while $v_{0}(x)$ and $F_{0}(x, u, s)$ are initial data.

From [19] one sees the equation 11.12 for $F$ can be solved allowing for the obtainment of $\tilde{\Sigma}$ as a function of the velocity gradient $\frac{\partial v}{\partial x}$. How this may be done is detailed in Appendix 1 (see Section 7.1); here below we summarize - for sake of clarity - the aforementioned calculations and give the algebraic expression of $\tilde{\Sigma}_{j 2}, j=1,2,3$.

The solution $F$ is obtained via the method of characteristics which allows for arbitrary initial data. One gets $F(x, t, u, s)=\sum_{k=1}^{+\infty} F_{k}(x, t, u) \sin \left(\frac{k \pi}{L} s\right)$, wherever

$$
\begin{aligned}
& F_{k}(x, t, u)=\frac{e^{-k^{2} \pi^{2} D t / L^{2}}}{\left\|\chi\left(\int_{0}^{t} \frac{\partial v}{\partial x}(x, \lambda) \mathrm{d} \lambda, u\right)\right\|^{3}} F_{0 k}\left(x, \frac{\chi\left(\int_{0}^{t} \frac{\partial v}{\partial x}(x, \lambda) \mathrm{d} \lambda, u\right)}{\left\|\chi\left(\int_{0}^{t} \frac{\partial v}{\partial x}(x, \lambda) \mathrm{d} \lambda, u\right)\right\|}\right) \\
& +\frac{\pi}{4} \frac{D}{L^{2}} a_{k} k^{2} \int_{0}^{t} \frac{e^{-k^{2} \pi^{2} D(t-\tau) / L^{2}}}{\left\|\chi\left(\int_{\tau}^{t} \frac{\partial v}{\partial x}(x, \lambda) \mathrm{d} \lambda, u\right)\right\|^{3}} \mathrm{~d} \tau
\end{aligned}
$$

with

$$
\chi(y, u)=\left(u_{1}-y u_{2}, u_{2}, u_{3}\right), \forall y \in \mathbb{R}, \forall u=\left(u_{1}, u_{2}, u_{3}\right) \in S_{2} .
$$


$F_{0 k}(x, u)$ is such that

$$
\begin{gathered}
F_{0}(x, u, s)=\sum_{k=1}^{+\infty} F_{0 k}(x, u) \sin \left(\frac{k \pi}{L} s\right) \\
1=\sum_{k=1}^{+\infty} a_{k} \sin \left(\frac{k \pi}{L} s\right), a_{k} \in \mathbb{R}
\end{gathered}
$$

Function $F$ allows for calculating $\tilde{\Sigma}_{j 2}, j=1,2,3$ in 1.11 as a function of the gradient $\frac{\partial v}{\partial x}(x, t)$. Observe that 1.8 now becomes the main equation, of unknown $v$. Assuming it has a solution $v$-actually the goal of the present paper is to prove the existence of such a solution - one gets the pressure field $p$ from $(1.9)$.

Next, one sees that for any $k \in \mathbb{N}^{*}$ function $F_{0 k}\left(x, u_{1}, u_{2}, u_{3}\right)$ - as a function of $u_{3}$ - depends on $u_{3}^{2}$, and so does $F_{k}$ for any $k$ as well. Consequently $\tilde{\Sigma}_{32}=0$ and $(1.10)$ always holds true.

In this work we shall assume that

$$
F_{0}=\frac{1}{4 \pi}
$$

which amounts to assuming $F_{0 k}=\frac{a_{k}}{4 \pi}, \forall k \in \mathbb{N}^{*}$. Use (1.17) into (1.14), and since $a_{k}=$ $\frac{2}{L} \int_{0}^{L} \sin \left(\frac{k \pi}{L} s\right) \mathrm{d} s=\frac{4}{k \pi}$ for any odd $k$ and $a_{k}=0$ for any even $k$, one formally gets

$$
F(x, t, u, s)=\frac{\tilde{a}(t, s)}{\left\|\chi\left(\int_{0}^{t} \frac{\partial v}{\partial x}(x, \lambda) \mathrm{d} \lambda, u\right)\right\|^{3}}-\int_{0}^{t} \frac{\frac{\partial \tilde{a}}{\partial t}(t-\tau, s)}{\left\|\chi\left(\int_{\tau}^{t} \frac{\partial v}{\partial x}(x, \lambda) \mathrm{d} \lambda, u\right)\right\|^{3}} \mathrm{~d} \tau
$$

where

$$
\tilde{a}(t, s)=\frac{1}{\pi^{2}} \sum_{p=1}^{+\infty} \frac{1}{2 p+1} e^{-D(2 p+1)^{2} \pi^{2} t / L^{2}} \sin \left(\frac{(2 p+1) \pi}{L} s\right), \forall t \geq 0, s \in(0, L)
$$

The assumption (1.17) is critical to ensure a K-BKZ type of equation in $v$ is obtained (see equation (1.23)); altering it would lead to a different kind of problem hence a different mathematical setting.

Posing $v(x, \tau)=0$ for $\tau<0$ leads to

$$
\int_{-\infty}^{0} \frac{\frac{\partial \tilde{a}}{\partial t}(t-s, s)}{\left\|\chi\left(\int_{\tau}^{t} \frac{\partial v}{\partial x}(x, \lambda) \mathrm{d} \lambda, u\right)\right\|^{3}} \mathrm{~d} \tau=-\frac{\tilde{a}(s, t)}{\left\|\chi\left(\int_{0}^{t} \frac{\partial v}{\partial x}(x, \lambda) \mathrm{d} \lambda, u\right)\right\|^{3}}
$$

and with help of 1.18

$$
F(x, t, u, s)=-\int_{-\infty}^{t} \frac{\frac{\partial \tilde{a}}{\partial t}(t-s, s)}{\left\|\chi\left(\int_{\tau}^{t} \frac{\partial v}{\partial x}(x, \lambda) \mathrm{d} \lambda, u\right)\right\|^{3}} \mathrm{~d} \tau
$$


which is equation 3.10 of [19] (reference in which the result is obtained via a Green's function technique), unanimously used when the IAA version theory is used. As an aside, we mention that in the rheology literature one commonly states that the "configurational probability density must vanish in the sufficiently distant past" i.e. $F_{0}=0$ for $t=-\infty$. But this gives $F_{0}=\frac{1}{4 \pi}$ for $t=0$, hence (1.17). However, taking $F_{0} \neq \frac{1}{4 \pi}$ leads to a different mathematical problem, unrelated to the fluid under scrutiny here.

Moreover, a straightforward calculation shows that:

$$
\frac{1}{L} \int_{0}^{L} \tilde{a}(t, s) \mathrm{d} s=\frac{2}{\pi^{3}} a_{\mathrm{DE}}(t)
$$

where the Doi-Edwards kernel (a.k.a. the Doi-Edwards relaxation function) $a_{\mathrm{DE}}$ is given by

$$
a_{\mathrm{DE}}(t)=\sum_{p=1}^{+\infty} \frac{1}{(2 p+1)^{2}} e^{-D(2 p+1)^{2} \pi^{2} t / L^{2}}, \forall t \geq 0
$$

Letting now $g_{\mathrm{DE}}: \mathbb{R} \rightarrow \mathbb{R}$

$$
g_{\mathrm{DE}}(y)=-\frac{2 \alpha}{\pi^{3}} \int_{S_{2}} \frac{u_{1} u_{2}}{\left[\left(u_{1}-u_{2} y\right)^{2}+u_{2}^{2}+u_{3}^{2}\right]^{3 / 2}} \mathrm{~d} u, \forall y \in \mathbb{R}
$$

we obtain

$$
\tilde{\Sigma}_{12}=-g_{\mathrm{DE}}\left(\int_{0}^{t} \frac{\partial v}{\partial x}(x, \tau) \mathrm{d} \tau\right) a_{\mathrm{DE}}(t)+\int_{0}^{t} g_{\mathrm{DE}}\left(\int_{\tau}^{t} \frac{\partial v}{\partial x}(x, r) \mathrm{d} r\right) a_{\mathrm{DE}}^{\prime}(t-\tau) \mathrm{d} \tau
$$

Before proceeding further, we make at this stage a final remark: had one chosen $F_{0} \neq \frac{1}{4 \pi}$, then equation (1.23) would have contained an additional term of the form $\frac{\partial \varphi}{\partial x}\left(t, \int_{0}^{t} \frac{\partial v}{\partial x}(x, r) \mathrm{d} r\right)$, where $\varphi$ is a "small" function whenever $\left(F_{0}-\frac{1}{4 \pi}\right)$ is "small", resulting in a different mathematical problem.

From the above considerations one infers the shear flow problem under scrutiny is tantamount to solving for $v$ the below integro-differential equation:

$$
\begin{aligned}
\frac{\partial v}{\partial t}= & -\frac{\partial}{\partial x} g_{\mathrm{DE}}\left(\int_{0}^{t} \frac{\partial v}{\partial x}(x, \tau) \mathrm{d} \tau\right) a_{\mathrm{DE}}(t) \\
& +\frac{\partial}{\partial x} \int_{0}^{t} g_{\mathrm{DE}}\left(\int_{\tau}^{t} \frac{\partial v}{\partial x}(x, r) \mathrm{d} r\right) a_{\mathrm{DE}}^{\prime}(t-\tau) \mathrm{d} \tau+f, t>0
\end{aligned}
$$

Now equation 1.23 - here obtained on molecular dynamics grounds - has been focused on within the area of viscoelastic fluids as it appears when one studies shear flows for the K-BKZ fluids. There is no contingency here as in their 1978 original paper [19], Doi and Edwards have shown the simplified IAA version of their nonlinear model actually enters the class of K-BKZ integral models, which are based on continuum mechanics concepts (for more on see [6], 32], [38]). Consequently, when undertaking the study of certain particular flows of the IAA-version of DE fluids one may capitalize on previously obtained results for K-BKZ liquids. 
In this paper we study equation (1.23) with more general functions $g$ and $a$ replacing $g_{\mathrm{DE}}$ and $a_{\mathrm{DE}}$, respectively. We prove a global in time solution existence result for small enough data. Equation (1.23) - as well as variants of it - was studied by various authors, see Renardy, Hrusa and Nohel [37], Engler [22], Brandon and Hrusa [8] and references cited therein.

The existence of local in time solutions [37] and of global solutions [22], 8] are known under more restrictive conditions compared to those stated in this paper. One of the assumptions in [22] and [8] is $g^{\prime}(y)<-\gamma$, for any $y \in \mathbb{R}$, with $\gamma>0$, which is not verified by the function $g=g_{\mathrm{DE}}$. This is a consequence of the fact that $g_{\mathrm{DE}}(0)=0, g_{\mathrm{DE}}^{\prime}(0)<0$ and $\lim _{y \rightarrow+\infty} g_{\mathrm{DE}}(y)=0$. Here we make use of the less restrictive assumption $g^{\prime}(y)<0$, for any $y \in[-\theta, \theta]$, with $\theta>0$ - assumption verified by $g_{\mathrm{DE}}$ - and show that the argument of $g^{\prime}$ is confined to $[-\theta, \theta]$. The requirement $g^{\prime}<0$ in a neighborhood of 0 is a necessary hyperbolicity condition for the solution local existence. For the work presented in this paper, this condition being valid only locally makes it necessary to control, w.r.t. time $t$, the argument $\int_{0}^{t} \frac{\partial v}{\partial x}(x, \tau) \mathrm{d} \tau$ of $g^{\prime}$. Observe that at a first sight, this argument may become large with increasing $t$; we obtain estimates for this term using the maximal function concept. Notice that in [22] the problem is studied in $n$-dimensional framework but in a weak functional setting which is unsuitable for our problem because the condition $g^{\prime}(y)<-\gamma, \forall y \in \mathbb{R}$, does not hold in our case.

Next, among the restrictive hypotheses invoked by the authors of [8] for function $a$ is that $a^{\prime \prime} \in L^{1}(0,+\infty)$, which $a=a_{\mathrm{DE}}$ does not verify. Comparatively, here we shall place significantly less restrictions on $a$ and accordingly will construct a class of totally monotone functions, an element of which is $a=a_{\mathrm{DE}}$. Moreover, we prove and make use of a new inversion formula for the operator $u \mapsto a * u$, a technique different from the (classical) one used in [8].

The manuscript is organized as following:

In Section 2 we introduce the problem and enunciate the main result.

Section 3 is devoted to the proof of several necessary results such as a Gårding type inequality and an inversion formula for the operator $u \mapsto a * u$.

In Section 4 we introduce an approximated problem and obtain useful estimates for its solution. The proof of the main result is achieved in Section 5 .

In the ending Section 6 we construct a class of totally monotone functions that is compatible with the hypothesis made about $a$.

\section{Presentation of the problem, of the main result, and the proof strategy.}

Let from now on $\Omega \subset \mathbb{R}$ be a bounded, open interval. Let the functions $f: \Omega \times[0,+\infty) \rightarrow \mathbb{R}$, $g: I \subset \mathbb{R} \rightarrow \mathbb{R}$, with $I \ni 0$ an open interval, $v_{0}: \Omega \rightarrow \mathbb{R}, a:[0,+\infty) \rightarrow \mathbb{R}$.

The aim is to search for a solution $v: \Omega \times[0,+\infty) \rightarrow \mathbb{R}$ to the below given initial boundary value problem:

$$
\begin{aligned}
& v_{t}(x, t)=-a(t) \frac{\partial}{\partial x} g\left(\int_{0}^{t} v_{x}(x, s) \mathrm{d} s\right)+\frac{\partial}{\partial x} \int_{0}^{t} g\left(\int_{s}^{t} v_{x}(x, \tau) \mathrm{d} \tau\right) a^{\prime}(t-s) \mathrm{d} s+f(x, t) \\
& v(x, t=0)=v_{0}(x), \forall x \in \Omega, \text { and } v(x, t)=0, \forall t<0 \\
& v=0, \forall x \in \partial \Omega, \forall t \geq 0
\end{aligned}
$$

In the above, $v_{x} \equiv \frac{\partial v}{\partial x}$ and $a^{\prime}$ stands for the derivative of $a$. Throughout this paper, any function defined for $t \geq 0$ is understood as being set equal to 0 for $t<0$, i.e. it has domain $\mathbb{R}$. 
Moreover, for a function $\varphi \in W^{k, 1}(0,+\infty)$ we denote by $\varphi^{(k)}$ the distributional derivative of $\varphi$ on $\mathbb{R}_{+}^{*}$, derivative which is understood to be extended to $\mathbb{R}$ by 0 . Define

$$
\bar{v}^{t}(x, s):=\int_{t-s}^{t} v(x, \tau) \mathrm{d} \tau, 0 \leq s, t ; x \in \Omega
$$

Equation (2.1) now takes on a simpler form:

$$
v_{t}(x, t)=\int_{0}^{+\infty} a^{\prime}(s) \frac{\partial}{\partial x} g\left(\bar{v}_{x}^{t}(x, s)\right) \mathrm{d} s+f(x, t)
$$

Drawing inspiration from [8], (2.4) can be re-written as

$$
v_{t}(x, t)+g^{\prime}(0) \int_{0}^{t} a(t-s) v_{x x}(x, s) \mathrm{d} s=f(x, t)+\mathcal{G}(x, t)
$$

where

$$
\begin{aligned}
\mathcal{G}(x, t) & =\int_{0}^{+\infty} a^{\prime}(s)\left[g^{\prime}\left(\bar{v}_{x}^{t}(x, s)\right)-g^{\prime}(0)\right] \bar{v}_{x x}^{t}(x, s) \mathrm{d} s \\
& =\int_{0}^{t} v_{x x}(x, s) \int_{t-s}^{+\infty} a^{\prime}(\tau)\left[g^{\prime}\left(\bar{v}_{x}^{t}(x, \tau)\right)-g^{\prime}(0)\right] \mathrm{d} \tau \mathrm{d} s
\end{aligned}
$$

Convolution with respect to $t$ is denoted as usually by $*$; therefore (2.5) can be re-written in a more close form as

$$
v_{t}+g^{\prime}(0) a * v_{x x}=f+\mathcal{G}
$$

We now proceed to presenting several constitutive assumptions. The function $g$ is taken such that:

$\left(g_{1}\right)$. there exist $\theta \in[0,1]$ and $K>0$, such that $g \in \mathscr{C}^{3}([-\theta, \theta], \mathbb{R})$ and $\left|g^{(3)}(y)-g^{(3)}(0)\right| \leq$ $K|y|, \forall y \in[-\theta, \theta]$

$\left(g_{2}\right) \cdot g(0)=g^{\prime \prime}(0)=0$

$\left(g_{3}\right) \cdot g^{\prime}(0)<0$

The function $f$ is such that

$\left(f_{1}\right) . f, f_{x}, f_{t} \in \mathscr{C}_{b}^{0}\left([0,+\infty) ; L^{2}(\Omega)\right) \cap L^{2}\left([0,+\infty) ; L^{2}(\Omega)\right)$,

$\left(f_{2}\right) . f_{t t} \in L^{2}\left([0,+\infty) ; L^{2}(\Omega)\right), \int_{0}^{t} f(x, s) \mathrm{d} s \in \mathscr{C}_{b}^{0}\left([0,+\infty) ; H^{1}(\Omega)\right)$,

where $\mathscr{C}_{b}^{0}([0,+\infty) ; X)$ is the set of all functions $w:[0,+\infty) \rightarrow X$ which are bounded and continous, and $X$ is a Banach space.

Next, let $v_{0}$ be such that

$\left(v_{0}\right)_{1} . v_{0} \in H^{2}(\Omega)$. 
We assume that $f$ and $v_{0}$ are compatible with the already stated initial-boundary conditions:

$$
v_{0}(x)=f(x, t=0)=0, \forall x \in \partial \Omega
$$

Let the measures associated to $f$ and $v_{0}$ be defined as:

$$
\begin{gathered}
F(f):=\sup _{t \geq 0} \int_{\Omega}\left[f^{2}+f_{x}^{2}+f_{t}^{2}+\left(\int_{0}^{t} f(x, s) \mathrm{d} s\right)^{2}+\left(\int_{0}^{t} f_{x}(x, s) \mathrm{d} s\right)^{2}\right] \mathrm{d} x \\
+\int_{0}^{+\infty} \int_{\Omega}\left(f^{2}+f_{x}^{2}+f_{t}^{2}+f_{t t}^{2}\right)(x, t) \mathrm{d} x \mathrm{~d} t \\
V_{0}\left(v_{0}\right)=\left\|v_{0}\right\|_{H^{2}(\Omega)}^{2}=\int_{\Omega}\left[v_{0}^{2}+\left(v_{0}^{\prime}\right)^{2}+\left(v_{0}^{\prime \prime}\right)^{2}\right](x) \mathrm{d} x
\end{gathered}
$$

For any function $\varphi \in L^{1}((0,+\infty))$ we denote by $\mathcal{F} \varphi$ (or alternatively by $\hat{\varphi}$ ) and $\mathcal{L} \varphi$ the corresponding Fourier and Laplace transforms, i.e.:

$$
\begin{gathered}
\mathcal{F} \varphi(\omega):=\int_{0}^{+\infty} \varphi(t) e^{-i \omega t} \mathrm{~d} t, \forall \omega \in \mathbb{R} \\
\mathcal{L} \varphi(z):=\int_{0}^{+\infty} \varphi(t) e^{-z t} \mathrm{~d} t, \forall z \in \mathbb{C}, \operatorname{Re} z \geq 0
\end{gathered}
$$

Let us now assume the function $a$ satisfies the below given hypotheses $\left(a_{1}\right)-\left(a_{5}\right)$ :

$\left(\mathrm{a}_{1}\right) . a \in W^{1,1}(0,+\infty), a^{\prime}(t) \leq 0$ a.e. $t \geq 0$,

There exists a sequence of functions $\left(a_{n}\right)_{n \in \mathbb{N}}, a_{n} \in \mathscr{C}^{2}\left([0,+\infty) \cap W^{2, \infty}([0,+\infty))\right.$ s.t.

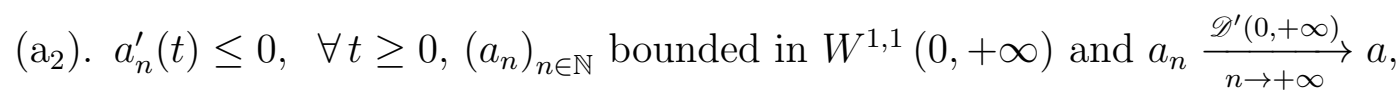

$\left(\mathrm{a}_{3}\right) \cdot \sup _{n \in \mathbb{N}}\left[\int_{0}^{1} t\left|a_{n}^{\prime \prime}(t)\right| \mathrm{d} t+\int_{1}^{+\infty} \sqrt{t}\left|a_{n}^{\prime \prime}(t)\right| \mathrm{d} t+\int_{1}^{+\infty} t^{2}\left|a_{n}^{\prime}(t)\right| \mathrm{d} t\right]<+\infty$

$\left(\mathrm{a}_{4}\right)$. there exist constants $M_{1}>0$ and $n_{0} \in \mathbb{N}$ s.t. $\operatorname{Re}\left(\mathcal{F} a_{n}(\omega)\right) \geq \frac{M_{1}}{1+\omega^{2}}, \forall n \in \mathbb{N}, n \geq n_{0}$, $\forall \omega \in \mathbb{R}$; observe that this is a strong positivity condition, common for this type of problems (see [8]).

(as). there exist constants $M_{2}>0, n_{0} \in \mathbb{N}, p \in \mathbb{N}^{*}, p \geq 2$, s.t. $\frac{\left[\mathcal{F}\left(a_{n}^{\prime}\right)\right]^{p}}{\mathcal{F} a_{n}} \in \mathcal{F}\left(B_{L^{1}(\mathbb{R})}\left(0, M_{2}\right)\right)$, $\forall n \in \mathbb{N}, n \geq n_{0}$, where $B_{L^{1}(\mathbb{R})}\left(0, M_{2}\right)$ denotes the ball in $L^{1}(\mathbb{R})$ centered at 0 and of radius $M_{2}$; this assumption will be used to obtain a representation for the solution $u$ of $a_{n} * u=b$ (see Theorem 3.1).

Remark 2.1. In Section 6 we shall construct a class of functions compliant with assumptions $\left(a_{1}\right)$ to $\left(a_{5}\right)$. This class contains the Doi-Edwards relaxation kernel $a_{D E}:[0,+\infty) \rightarrow \mathbb{R}$,

$$
a_{D E}(t)=\sum_{k \geq 1} \frac{1}{(2 k+1)^{2}} e^{-(2 k+1)^{2} \pi^{2} D t / L^{2}}
$$

Also, since $g_{D E} \in \mathscr{C}^{\infty}(\mathbb{R})$ is an odd function and $g_{D E}^{\prime}(0)=-\frac{6 \alpha}{\pi^{3}} \int_{S_{2}} u_{1}^{2} u_{2}^{2} \mathrm{~d} u<0$, then $g_{D E}$ also verifies $\left(g_{1}\right)-\left(g_{3}\right)$ and this paper results equally apply to the function $g_{D E}$. 
The main result of this paper is stated below:

Theorem 2.1 (Main Result). Assume that the hypotheses on the data given in $\left(g_{1}\right)-\left(g_{3}\right),\left(f_{1}\right)$ $\left(f_{2}\right),\left(v_{0}\right)_{1},\left(a_{1}\right)-\left(a_{5}\right)$ and (2.8) hold true. Then there exists a $\delta>0$ such that, if the additional smallness assumption $F(f)+V_{0}\left(v_{0}\right) \leq \delta$ is verified, then there exists at least a solution

$$
v \in\left\{\bigcap_{m=0}^{2} W^{m, \infty}\left((0,+\infty) ; H^{2-m}(\Omega)\right)\right\} \cap\left\{\bigcap_{m=0}^{2} W^{m, 2}\left((0,+\infty) ; H^{2-m}(\Omega)\right)\right\}
$$

with

$$
\int_{0}^{t} v(x, s) \mathrm{d} s \in L^{\infty}\left((0,+\infty) ; H^{3}(\Omega)\right)
$$

to the problem (2.4), 2.2)-(2.3).

Next we take on to introducing - and explaining - the proof stages for the aforementioned Theorem 2.1. In short, first we obtain a regularized problem $\left(P_{n}\right)$ obtained from (2.5) with $a$ being replaced by a sequence $a_{n}$ satisfying hypotheses $\left(a_{1}\right)$ to $\left(a_{5}\right)$. Doing this allows to obtain a local in time existence and uniqueness result capitalizing on Renardy's result in [37]. Next goal is to obtain estimates independent of $n$ granting the global existence of the solution for the approximated problem $\left(P_{n}\right)$ and in the end, letting $n \rightarrow+\infty$, obtaining our result. How to get these estimates is explained below.

Let $u(x, t)=\int_{0}^{t} v(x, \tau) \mathrm{d} \tau$. For any $t>0$, let $\mathcal{E}(t)$ stand for the sum of squared $L_{t}^{\infty} L_{x}^{2}$ norms of all derivatives in $x$ and $t$ of $u$ up to third order and of all squared $L_{t}^{2} L_{x}^{2}$ norms of all derivatives in $x$ and $t$ of $v$ up to second order (see (4.4)). We prove that if $\mathcal{E}(t)$ is "small" for $t$ close to 0 (a consequence of the assumption made on data $v_{0}$ and $f$ ), then $\mathcal{E}(t)$ stays "small" for any $t$. We do this by obtaining an inequality of the type

$$
\mathcal{E}(t) \leq \frac{1}{2} \mathcal{E}(t)+\text { "small enough" quantities depending uniquely on } V_{0} \text { and } F
$$

To achieve that, we calculate three energy estimates (in a way similar in nature with that of Brandon and Hrusa [8]: we derivate (2.7) $i$-times (with $i \in\{0,1,2\}$ ) w.r.t. time $t$, then multiply the result by $\frac{d^{i} v}{d t^{i}}$ and integrate on $Q_{t}:=\Omega \times(0, t)$. To calculate the second order derivative one uses a finite difference operator $\triangle_{h} w(t)=w(t+h)-w(t)$, see (3.2). We sum up the resulting three equations and get an equality in which the most important term originates from the convolution part in the lhs of (2.7). This term reads

$$
-g^{\prime}(0)\left[Q\left(v_{x}, t, a\right)+Q\left(v_{x t}, t, a\right)+Q\left(v_{x t t}, t, a\right)\right]
$$

where $Q(w, t, a)=\int_{0}^{t} \int_{\Omega} w(x, s)(a * w)(x, s) \mathrm{d} x \mathrm{~d} s$ (see (3.1)). We lower bound 2.14) using a Gårding type inequality: see Lemma 3.1 .

The terms denoted by $\mathcal{G}$ in 2.7 can be controlled w.r.t. well chosen norms by carrying out an integration by parts w.r.t. time $t$, switching the time derivatives onto $a$ and using the fact that $t a^{\prime \prime} \in L^{1}(0,1)$ (see assumption $\left(a_{3}\right)$ ). This allows to upper bound the $L_{t}^{\infty} L_{x}^{2}$ norms of $v, v_{x}, v_{t}, v_{x t}, v_{t t}$, and the $L_{t}^{2} L_{x}^{2}$ norms of $v, v_{x}, v_{t}, v_{x t}$. The results are gathered into $\mathcal{E}_{1}$, see (4.6). We point out that the aforementioned energy estimates do not provide norm estimates for $v_{x x}$. To cope with this difficulty we use (2.7) which allows to express $v_{x x}$ as a function of $v_{t}, f$ and $\mathcal{G}$ with the help of an inversion Theorem for the operator $w \mapsto a * w$ and using the 
previously obtained estimates. We cannot use the resolvent kernel technique like in Brandon and Hrusa [9] because in this paper case $r^{\prime} \notin L^{1}(\mathbb{R})$ (as $a^{\prime \prime} \notin L^{1}\left(\mathbb{R}_{+}\right)$). Because of that we prove a point-wise inversion Theorem for the convolution of $a$ assuming the rather pretty weak constraints $\left(a_{1}\right)-\left(a_{5}\right)$ on $a$ : see Theorem 3.1.

\section{Preliminaries.}

For any $T>0, w \in \mathscr{C}^{0}\left([0, T] ; L^{2}(\Omega)\right), b \in L^{1}(0,+\infty)$ and $t \in[0, T]$, we define

$$
\begin{aligned}
Q(w, t, b) & :=\int_{0}^{t} \int_{\Omega} w(x, s) \int_{0}^{s} b(s-\tau) w(x, \tau) \mathrm{d} \tau \mathrm{d} x \mathrm{~d} s \\
& =\int_{0}^{t} \int_{\Omega} w(x, s)(b * w)(x, s) \mathrm{d} x \mathrm{~d} s
\end{aligned}
$$

where $w$ is considered as extended by 0 on $(T,+\infty)$. For any $T>0$ and $h \in(0, T)$, we define the finite difference operator $\Delta_{h}$

$$
\left(\Delta_{h} w\right)(x, t)=w(x, t+h)-w(x, t)
$$

as a linear operator from $\mathscr{C}^{0}\left([0, T-h] ; L^{2}(\Omega)\right)$ onto $\mathscr{C}^{0}\left([0, T] ; L^{2}(\Omega)\right)$.

Moreover, if $X(J)$ denotes a space of functions defined on $J \subset \mathbb{R}$ and $I \subset J$, then $X_{I}(J)$ stands for the subspace of functions $X(J)$ the supports of which are included in $I$ (i.e. that vanish on $J-I)$.

Recall that $b \in L^{1}\left(\mathbb{R}_{+}\right)$is of positive type if, for any $t \geq 0$ and any $\varphi \in L^{2}\left(\mathbb{R}_{+}\right)$, it satisfies $\int_{0}^{t} \varphi(s) \int_{0}^{s} b(s-\tau) \varphi(\tau) \mathrm{d} \tau \mathrm{d} s \geq 0$. Next, $b$ is said to be of strong positive type if there exists $\epsilon>0$ s.t. the function $b(t)-\epsilon e^{-t}$ is of positive type. Moreover, $Q_{t}:=\Omega \times(0, t)$.

The following Lemma is a Gårding type inequality with boundary terms. It is proved in [9] using preliminary results due to Staffans [39] (see also [23] and [41]). Here we shorten the original proof of [9] and remove the extraneous assumptions $b \in W^{3,1}(0,+\infty), b^{\prime \prime} \geq 0$.

Lemma 3.1. Assume $b \in L_{\mathbb{R}_{+}}^{1}(\mathbb{R})$ is such that $\operatorname{Re}(\mathcal{F} b(\omega)) \geq \frac{M_{1}}{1+\omega^{2}}$, for any $\omega \in \mathbb{R}$, where $M_{1}>0$. Then, for any $T>0, w \in \mathscr{C}^{1}\left([0, T], L^{2}(\Omega)\right)$ and $t \in[0, T)$, we have

$$
\begin{aligned}
& \int_{\Omega} w^{2}(x, t) \mathrm{d} x+\int_{0}^{t} \int_{\Omega} w^{2}(x, s) \mathrm{d} x \mathrm{~d} s \\
& \leq C\left[\frac{1}{M_{1}} Q(w, t, b)+\frac{1}{M_{1}} Q\left(w_{t}, t, b\right)+\int_{\Omega} w^{2}(x, 0) \mathrm{d} x\right]
\end{aligned}
$$

with $C>0$ independent of $T, t, w$ and $b$.

Moreover, if $w \in \mathscr{C}^{0}\left([0, T], L^{2}(\Omega)\right)$, then, for any $t \in[0, T]$,

$$
\begin{aligned}
& \int_{\Omega} w^{2}(x, t) \mathrm{d} x+\int_{0}^{t} \int_{\Omega} w^{2}(x, s) \mathrm{d} x \mathrm{~d} s \\
& \leq C\left[\frac{1}{M_{1}} Q(w, t, b)+\frac{1}{M_{1}} \liminf _{h \rightarrow 0_{+}} \frac{1}{h^{2}} Q\left(\triangle_{h} w, t, b\right)+\int_{\Omega} w^{2}(x, 0) \mathrm{d} x\right]
\end{aligned}
$$


Proof. Assuming that inequality (3.3) holds true, we undertake to proving (3.4). Let $w \in$ $\mathscr{C}^{0}\left([0, T], L^{2}(\Omega)\right)$ and $t \in[0, T)$ be fixed. For $0<h<(T-t) / 2$, define the function $w_{h} \in$ $\mathscr{C}^{1}\left([0,(t+T) / 2], L^{2}(\Omega)\right)$ by

$$
w_{h}(s):=\frac{1}{h} \int_{s}^{s+h} w(\sigma) \mathrm{d} \sigma, s \in[0,(t+T) / 2)
$$

Applying (3.3) to $w_{h}$ and passing to the limit $\liminf _{h \rightarrow 0_{+}}$gives 3.4 .

We now prove $(3.3)$. Let $w \in \mathscr{C}^{1}\left([0, t], L^{2}(\Omega)\right), t \in[0, T)$ be fixed, and let $\tilde{w} \in L_{[0, t)}^{2}\left(\mathbb{R}, L^{2}(\Omega)\right)$ be defined by $\tilde{w}=w$ a.e. in $[0, t]$ and $\tilde{w}=0$ outside. Denote by $D \tilde{w}$ the distributional derivative of $\tilde{w}$ and by $\tilde{w}^{\prime}$ its regular part, i.e.

$$
D \tilde{w}=\tilde{w}^{\prime}+w(0) \delta_{0}-w(t) \delta_{t}
$$

Due to the Parseval identity we have

$$
Q(w, t, b)=\frac{1}{2 \pi} \int_{\mathbb{R}} \int_{\Omega} \operatorname{Re}(\hat{b}(\tau))|\widehat{\tilde{w}}(x, \tau)|^{2} \mathrm{~d} x \mathrm{~d} \tau
$$

and a similar equation with $w^{\prime}$ instead of $w$ as well. For $\lambda>0$ (to be later determined) define $I(w)$ by

$$
I(w):=Q\left(\tilde{w}^{\prime}, t, b\right)+\lambda Q(\tilde{w}, t, b)+\frac{3 M_{1}}{2} \int_{\Omega} w^{2}(x, 0) \mathrm{d} x
$$

By (3.6) and (3.7) and the strong positivity of $b$,

$$
\begin{aligned}
& I(w) \geq \\
& \frac{M_{1}}{2 \pi} \int_{\mathbb{R}} \int_{\Omega}\left(\left|i \tau \widehat{\widetilde{w}}(\tau)+w(t) e^{-i \tau t}-w(0)\right|^{2}+\lambda|\widehat{\widetilde{w}}(\tau)|^{2}+3|w(0)|^{2}\right) \mathrm{d} x \frac{\mathrm{d} \tau}{1+\tau^{2}}
\end{aligned}
$$

Since for any $(a, b, c) \in \mathbb{C}^{3}$ we have $|a+b+c|^{2} \geq \frac{|a|^{2}+|b|^{2}}{2}-2|a||b|-3|c|^{2}$, inequality (3.9) implies

$$
\begin{aligned}
& I(w) \geq \\
& \frac{M_{1}}{2 \pi} \int_{\mathbb{R}} \int_{\Omega}\left(\frac{|\tau|^{2}+2 \lambda}{2}|\widehat{\tilde{w}}(\tau)|^{2}+|w(t)|^{2} \beta \sqrt{|\tau|}-2|w(t)||\tau||\widehat{\tilde{w}}(\tau)|\right) \mathrm{d} x \frac{\mathrm{d} \tau}{1+\tau^{2}}
\end{aligned}
$$

with

$$
\beta=\frac{1}{2}\left(\int_{\mathbb{R}} \frac{\mathrm{d} \tau}{1+\tau^{2}}\right) /\left(\int_{\mathbb{R}} \frac{\sqrt{|\tau|}}{1+\tau^{2}} \mathrm{~d} \tau\right)
$$

But:

$$
\begin{aligned}
2|w(t)||\tau||\widehat{\tilde{w}}(\tau)| & \leq \frac{\beta}{2} \sqrt{|\tau|}|w(t)|^{2}+\frac{2}{\beta}|\tau|^{3 / 2}|\widehat{\tilde{w}}(\tau)|^{2} \\
& \leq \frac{\beta}{2} \sqrt{|\tau|}|w(t)|^{2}+\left(\frac{|\tau|^{2}}{4}+L\right)|\widehat{\tilde{w}}(\tau)|^{2}
\end{aligned}
$$

with $L>0$ independent of $t, w, b$. Choose $\lambda=L+1 / 4$. By 3.10 and 3.12 we get 


$$
I(w) \geq \frac{M_{1}}{2 \pi} \int_{\mathbb{R}} \int_{\Omega}\left(\frac{|\tau|^{2}+1}{4}|\widehat{\tilde{w}}(\tau)|^{2}+\frac{\beta \sqrt{|\tau|}}{2}|w(t)|^{2}\right) \mathrm{d} x \frac{\mathrm{d} \tau}{1+\tau^{2}}
$$

which is $(3.3)$.

We now prove that, under suitable assumptions application $w \mapsto b * w$ is invertible, and obtain an inversion formula. We use truncated Neumann series and a special assumption (see $\left(b_{3}\right)$ below) in order to control the remainder term.

For $b \in L^{1}(\mathbb{R})$, let the $k$-times convolution de denoted as $b^{* k}:=\underbrace{b * b * \cdots * b}_{k \text { times }}$. For $1 \leq q \leq$ $+\infty$ and $t_{0} \in(0,+\infty]$, the mapping $\mathcal{R}_{t_{0}, q}$ is defined by:

$$
\mathcal{R}_{t_{0}, q}: \begin{cases}L_{\left[0, t_{0}\right)}^{q}\left(-\infty, t_{0}\right) & \longrightarrow W_{\left[0, t_{0}\right)}^{1, q}\left(-\infty, t_{0}\right) \\ w & \mapsto b * w\end{cases}
$$

Here $b * w(t):=\int_{0}^{t} b(t-s) w(s) \mathrm{d} s$, for any $t<t_{0}$. We always write $\mathcal{R}$ in place of $\mathcal{R}_{+\infty, 2}$.

Next, function $b$ is assumed to comply with:

$\left(b_{1}\right) b \in W^{1,1}(0,+\infty), b\left(0_{+}\right) \neq 0$,

$\left(\mathrm{b}_{2}\right)$ there exists $M>0, \beta>0$ s.t.

$$
|\mathcal{L} b(z)| \geq \frac{M}{1+|z|^{\beta}}, \forall z \in \mathbb{C}, \operatorname{Re}(z) \geq 0
$$

$\left(\mathrm{b}_{3}\right)$ there exists $p \in \mathbb{N}^{*}, p \geq 2$ s.t.

$$
\mathcal{F}^{-1}\left[\frac{\left(\mathcal{F} b^{\prime}\right)^{p}}{\mathcal{F} b}\right] \in L^{1}(\mathbb{R})
$$

Our goal is to prove the following inversion Theorem:

Theorem 3.1 (Inversion Theorem). Let the assumptions $\left(b_{1}\right)-\left(b_{3}\right)$ hold true. Then:

(i) for any $1 \leq q \leq+\infty$ and $t_{0} \in(0,+\infty]$, the mapping $\mathcal{R}_{t_{0}, q}$ is a Banach isomorphism;

(ii) functions $B_{1}, B_{2}$ that depend only on $b$ and are being given by

$$
\begin{gathered}
B_{1}=\sum_{k=1}^{p-1}(-1)^{k} \frac{\left(b^{\prime}\right)^{* k}}{b^{k+1}\left(0_{+}\right)} \\
B_{2}=\frac{(-1)^{p}}{b^{p}\left(0_{+}\right)} \mathcal{F}^{-1}\left[\frac{\left(\mathcal{F} b^{\prime}\right)^{p}}{\mathcal{F} b}\right],
\end{gathered}
$$

belong to $L_{\mathbb{R}_{+}}^{1}(\mathbb{R})$;

(iii) for any $l \in W_{\left[0, t_{0}\right)}^{1, q}\left(-\infty, t_{0}\right)$, one has

$$
\mathcal{R}_{t_{0}, q}^{-1}(l)=\frac{l^{\prime}}{b\left(0_{+}\right)}+B_{1} * l^{\prime}+B_{2} * l
$$


For the proof we first need to introduce and prove two preliminary Lemmas.

Lemma 3.2. Assume that $b \in W^{1,1}\left(\mathbb{R}_{+}^{*}\right), b\left(0_{+}\right) \neq 0$. Let $1 \leq q \leq+\infty, t_{0} \in(0,+\infty)$. Then $\mathcal{R}_{t_{0}, q}$ is a continuous injection.

Proof. We begin by showing $\mathcal{R}_{t_{0}, q}$ is well defined and continous. Since $b \in W^{1,1}\left(\mathbb{R}_{+}^{*}\right)$, it is clear that for any $w \in L_{\left[0, t_{0}\right)}^{q}\left(-\infty, t_{0}\right)$, the function $b * w$ belongs to $W_{\left[0, t_{0}\right)}^{1, q}\left(-\infty, t_{0}\right)$. Moreover, $(b * w)^{\prime}=\left[b\left(0_{+}\right) w+b^{\prime} * w\right]$. Hence

$$
\left\|\mathcal{R}_{t_{0}, q}(w)\right\|_{W^{1, q}\left(0, t_{0}\right)} \leq\left[\left|b\left(0_{+}\right)\right|+\|b\|_{W^{1,1}\left(\mathbb{R}_{+}^{*}\right)}\right]\|w\|_{L^{q}\left(0, t_{0}\right)}
$$

which proves $\mathcal{R}_{t_{0}, q}$ is indeed continous.

Next, assume $w \in L_{\left[0, t_{0}\right)}^{q}\left(-\infty, t_{0}\right)$ satisfies $\mathcal{R}_{t_{0}, q}(w)=0$. Derivating the later leads to

$$
w(s)+\int_{0}^{s} \frac{b^{\prime}(s-\tau)}{b\left(0_{+}\right)} w(\tau) \mathrm{d} \tau=0, \text { a.e. } s<t_{0}
$$

Multiply 3.20 by $e^{-\theta s}, \theta>0$, and set $w_{1}(s)=e^{-\theta s} w(s), b_{1}(s)=\frac{b^{\prime}(s)}{b\left(0_{+}\right)} e^{-\theta s}$. Equality 3.20 can now be re-written as

$$
w_{1}(s)+\int_{0}^{t} b_{1}(s-\tau) w_{1}(\tau) \mathrm{d} \tau=0, \text { a.e. } s<t_{0}
$$

It implies that

$$
\left\|w_{1}\right\|_{L^{q}\left(0, t_{0}\right)} \leq\left\|b_{1}\right\|_{L^{1}\left(\mathbb{R}_{+}^{*}\right)}\left\|w_{1}\right\|_{L^{q}\left(0, t_{0}\right)}
$$

Notice that $\left\|b_{1}\right\|_{L^{1}}=\int_{0}^{+\infty} e^{-\theta s} \frac{\left|b^{\prime}(s)\right|}{\left|b\left(0_{+}\right)\right|} \mathrm{d} s \underset{\theta \rightarrow+\infty}{\longrightarrow} 0$. Pick up a $\theta>0$ large enough s.t.

$\left\|b_{1}\right\|_{L^{1}\left(\mathbb{R}_{+}^{*}\right)}<1$. From 3.22 we get $\left\|w_{1}\right\|_{L^{1}(0, t)}=0$. Finally $w=0$ and $\mathcal{R}_{t_{0}, q}$ is an injection mapping.

Lemma 3.3. The Theorem 3.1 holds true for $t_{0}=+\infty$ and $q=2$.

Proof. The proof consists of four steps.

\section{Step 1.}

First we prove the existence, as a consequence of assumptions $\left(b_{1}\right)-\left(b_{2}\right)$ on $b$, of a constant $M_{3}>0$ s.t.

$$
|\mathcal{F}(b)(\omega)| \geq \frac{M_{3}}{1+|\omega|}, \forall \omega \in \mathbb{R}
$$

Indeed, one sees that $\left(b_{2}\right)$ implies

$$
|\mathcal{F}(b)(\omega)| \geq \frac{M}{1+|\omega|^{\beta}}, \forall \omega \in \mathbb{R}
$$

Then it suffices to prove the existence of $m_{1}, m_{2}>0$ s.t. $|\mathcal{F}(b)(\omega)| \geq \frac{m_{1}}{|\omega|}, \forall \omega \in \mathbb{R}$ with $|\omega| \geq m_{2}$. This follows from the fact that $|\mathcal{F}(b)(\omega)|=\frac{1}{i \omega}\left[\mathcal{F}\left(b^{\prime}\right)(\omega)+b\left(0_{+}\right)\right]$, that $\mathcal{F}\left(b^{\prime}\right)(\omega) \rightarrow 0$ for $|\omega| \rightarrow \infty$, and $b\left(0_{+}\right) \neq 0$.

\section{Step 2.}


Now we prove $\mathcal{R}$ is a Banach isomorphism. Due to Lemma 3.2, one only needs to prove $\mathcal{R}$ is surjective. To begin with, one establishes that, for any $w \in L_{\mathbb{R}_{+}}^{2}(\mathbb{R})$, one has (with $M_{3}>0$ the constant in (3.23)

$$
\|w\|_{L^{2}(\mathbb{R})} \leq \frac{1}{\sqrt{\pi} M_{3}}\|\mathcal{R}(w)\|_{H^{1}(\mathbb{R})}
$$

Actually using Parseval's identity and (3.23) one gets

$$
\sqrt{2 \pi}\|w\|_{L^{2}(\mathbb{R})}=\|\mathcal{F} w\|_{L^{2}(\mathbb{R})}=\left\|\frac{\mathcal{F} \mathcal{R}(w)}{\mathcal{F} b}\right\|_{L^{2}(\mathbb{R})} \leq \frac{1}{M_{3}}\|(1+|\omega|) \mathcal{F} \mathcal{R}(w)\|_{L^{2}(\mathbb{R})}
$$

Since $(1+|\omega|) \leq \sqrt{2\left(1+\omega^{2}\right)}$, inequality (3.25) implies inequality (3.24). Next, inequalities (3.19) and (3.24) prove that $\mathcal{R}\left(L_{\mathbb{R}_{+}}^{2}(\mathbb{R})\right)$ is closed. Therefore, in order to prove that $\mathcal{R}$ is surjective it is sufficient to show that the dense subset $\left(\mathscr{C}_{c}^{\infty}\right)_{(0,+\infty)}(\mathbb{R})$ of $H_{\mathbb{R}_{+}}^{1}(\mathbb{R})$ is included in $\mathcal{R}\left(L_{\mathbb{R}_{+}}^{2}(\mathbb{R})\right)$.

Let $r \in\left(\mathscr{C}_{c}^{\infty}\right)_{(0,+\infty)}(\mathbb{R})$. We search for $w \in L_{\mathbb{R}_{+}}^{2}(\mathbb{R})$ s.t. $b * w=r$. Since we are unable to identify the support of $w$ by Fourier transform, we use Laplace transform instead. Consider the function

$$
z \in\{z \in \mathbb{C} / \operatorname{Re}(z) \geq 0\} \mapsto \frac{\mathcal{L} r(z)}{\mathcal{L} b(z)} \in \mathbb{C}
$$

which is well defined based on $\left(b_{2}\right)$ and the fact that $r \in\left(\mathscr{C}_{c}^{\infty}\right)_{(0,+\infty)}(\mathbb{R})$. This function is clearly continuous on $\operatorname{Re}(z) \geq 0$ and analytic on $\operatorname{Re}(z)>0$. As for any $z \in \mathbb{C}$ and $\gamma \in \mathbb{N}$, $\mathcal{L} r^{(\gamma)}(z)=z^{\gamma} \mathcal{L} r(z)$, and as $r^{(\gamma)} \in L^{1}(\mathbb{R})$, we deduce that there exists $m_{3} \geq 0$ s.t.

$$
|\mathcal{L} r(z)| \leq \frac{m_{3}}{1+|z|^{\beta+2}}, \forall z \in \mathbb{C}, \operatorname{Re}(z) \geq 0
$$

Now it easily follows the existence of $m_{4} \geq 0$ s.t.

$$
\left|\frac{\mathcal{L} r(z)}{\mathcal{L} b(z)}\right| \leq \frac{m_{4}}{1+|z|^{2}}, \forall z \in \mathbb{C}, \operatorname{Re}(z) \geq 0
$$

Next, with the help of Bromwich-Mellin formula, for any $t \in \mathbb{R}$ and for fixed $x>0$, define $w$ as

$$
w(t):=\frac{1}{2 \pi i} \int_{\mathbb{R}} e^{t(x+i y)} \frac{\mathcal{L} r}{\mathcal{L} b}(x+i y) \mathrm{d} y
$$

Owing to Cauchy's formula and invoking (3.26), $w$ thus defined is independent of $x>0$. Also, for fixed $t<0$, letting $x \rightarrow+\infty$ in (3.27) leads to $w(t)=0$. This is $w(t)=0$ for any $t<0$. Next, for any fixed $t \in \mathbb{R}$, using Lebesgue's Theorem we calculate the limit for $x \rightarrow 0$ of (3.27) and obtain $w=\mathcal{F}^{-1}\left(\frac{\mathcal{F} r}{\mathcal{F} b}\right)$. By Parseval's identity and by $(3.26), w$ is clearly an element of $L_{\mathbb{R}_{+}}^{2}(\mathbb{R})$ and satisfies $\mathcal{R}(w)=r$. Therefore $\mathcal{R}$ is surjective.

\section{Step 3.}

The task now is proving the representation formula. Let $w \in L_{\mathbb{R}_{+}}^{2}(\mathbb{R})$ and set $l=\mathcal{R}(w)$. Derivation of the later gives

$$
w+\frac{b^{\prime}}{b\left(0_{+}\right)} * w=\frac{l^{\prime}}{b\left(0_{+}\right)}
$$


Convolute (3.28) with the operator $\sum_{k=0}^{p-1}(-1)^{k}\left(\frac{b^{\prime}}{b\left(0_{+}\right)}\right)^{* k} *\left(\right.$ by convention $\left.\left(\frac{b^{\prime}}{b\left(0_{+}\right)}\right)^{* 0}=\delta_{0}\right)$. We obtain:

$$
w=\frac{l^{\prime}}{b\left(0_{+}\right)}+\left(B_{1} * l^{\prime}\right)+\frac{(-1)^{p}}{b^{p}\left(0_{+}\right)}\left[\left(b^{\prime}\right)^{* p} * w\right]
$$

Since $l=b * w$, we get $\mathcal{F} l=\mathcal{F} b \mathcal{F} w$. Hence

$$
\mathcal{F}\left[\left(b^{\prime}\right)^{* p} * w\right]=\left(\mathcal{F} b^{\prime}\right)^{p} \frac{\mathcal{F} l}{\mathcal{F} b}
$$

By hypothesis $\left(b_{3}\right), \frac{\left(\mathcal{F} b^{\prime}\right)^{p}}{\mathcal{F} b} \in L^{\infty}(\mathbb{R})$, which proves that inequality 3.30 holds in $L^{2}(\mathbb{R})$ since $\mathcal{F} l \in L^{2}(\mathbb{R})$. This fact allows to state that $\frac{\left(-b^{\prime}\right)^{* p}}{b^{p}\left(0_{+}\right)} * w=B_{2} * l$ with $B_{2}$ given by (3.17). Now, for any $w \in L_{\mathbb{R}_{+}}^{2}(\mathbb{R})$ and $l=\mathcal{R}(w), 3.29$ gives the representation formula

$$
w=\frac{l^{\prime}}{b\left(0_{+}\right)}+B_{1} * l^{\prime}+B_{2} * l
$$

\section{Step 4.}

Let us now show that the support of $B_{1}$ and that of $B_{2}$ are included in $\mathbb{R}_{+}$.

Since the support of $b^{\prime}$ is in $\mathbb{R}_{+}, B_{1}$ also has its support in $\mathbb{R}_{+}$due to formula $(3.16)$. Let $\rho \in \mathscr{D}_{\mathbb{R}_{+}}(\mathbb{R})$ and set $w=\mathcal{R}^{-1}(\rho)$ (see Step 2.). Equation (3.31) now ensures that, a.e. $t<0$,

$$
0=w(t)=\frac{\rho^{\prime}(t)}{b\left(0_{+}\right)}+\left(B_{1} * \rho^{\prime}\right)(t)+\left(B_{2} * \rho\right)(t)
$$

Since $\rho^{\prime}(s)=0$ a.e. $s<0$ and since $B_{1}$ has support in $\mathbb{R}_{+}$, we get

$$
\left(B_{2} * \rho\right)(t)=0 \text {, a.e. } t<0
$$

Take $\rho \geq 0, \rho \neq 0$, and set $\rho_{n}(t)=n \rho(n t), n \in \mathbb{N}^{*}, t \in \mathbb{R}$. We know that:

$$
B_{2} * \rho_{n} \underset{n \rightarrow+\infty}{\stackrel{L^{1}(\mathbb{R})}{\longrightarrow}}\|\rho\|_{L^{1}} B_{2}
$$
in $\mathbb{R}_{+}$.

Taking $\rho=\rho_{n}$ in 3.33 and using (3.34) we obtain $B_{2}=0$ a.e. $t<0$. Hence $B_{2}$ has support

We are now in a position allowing to prove the previously stated Inversion Theorem 3.1.

Proof. Proof of the Inversion Theorem 3.1

Let $q \in[1,+\infty)$ and $t_{0} \in \mathbb{R}_{+}^{*} \cup\{+\infty\}$. Define the mapping $\mathcal{S}_{t_{0}, q}$ by:

$$
\mathcal{S}_{t_{0}, q}= \begin{cases}W_{\left[0, t_{0}\right)}^{1, q}\left(-\infty, t_{0}\right) & \longrightarrow L_{\left[0, t_{0}\right)}^{q}\left(-\infty, t_{0}\right) \\ l & \mapsto \frac{l^{\prime}}{b\left(0_{+}\right)}+B_{1} * l^{\prime}+B_{2} * l\end{cases}
$$

with $B_{1}, B_{2} \in L_{\mathbb{R}_{+}}^{1}(\mathbb{R})$ given by 3.16 - 3.17$)$. Clearly $\mathcal{S}_{t_{0}, q}$ is well defined and continuous.

We begin by studying the case $t_{0}=+\infty$.

Notice that $\mathcal{S}_{+\infty, q} \circ \mathcal{R}_{+\infty, q}$ restricted to $D=L_{\mathbb{R}_{+}}^{q}(\mathbb{R}) \cap L_{\mathbb{R}_{+}}^{2}(\mathbb{R})$ is the identity (see Lemma 3.3). Since $D$ is dense in $L_{\mathbb{R}_{+}}^{q}(\mathbb{R})$, and $\mathcal{S}_{+\infty, q}$ and $\mathcal{R}_{+\infty, q}$ are continuous, we find that $\mathcal{S}_{+\infty, q} \circ \mathcal{R}_{+\infty, q}$ is the identity on $L_{\mathbb{R}_{+}}^{q}(\mathbb{R})$. Similarly, $\mathcal{R}_{+\infty, q} \circ \mathcal{S}_{+\infty, q}$ is the identity on $W_{\mathbb{R}_{+}}^{1, q}(\mathbb{R})$. This proves the Theorem for $t_{0}=+\infty$. 
Assume now that $t_{0}>0$ and $q \in[1,+\infty]$. We know from Lemma 3.2 that $\mathcal{R}_{t_{0}, q}$ is continuous and injective. We now prove that $\mathcal{R}_{t_{0}, q}$ is surjective and that $\mathcal{S}_{t_{0}, q}$ is its inverse. Let $l \in$ $W_{\left[0, t_{0}\right)}^{1, q}\left(-\infty, t_{0}\right)$ and extend $l$ into $L \in W_{\left[0,2 t_{0}\right)}^{1, q}(\mathbb{R})$ by reflexion

$$
L(t)= \begin{cases}l(t) & \text { for } t<t_{0} \\ l\left(2 t_{0}-t\right) & \text { for } t>t_{0}\end{cases}
$$

Let $W=\left(\mathcal{S}_{+\infty, q}\right)(L)$ and define $w \in L_{\left[0, t_{0}\right)}^{q}\left(-\infty, t_{0}\right)$ as the restriction of $W$ to $\left(-\infty, t_{0}\right)$. Then, $b * w=b * W=l$ on $\left(-\infty, t_{0}\right)$, and:

$$
w=W=\frac{L^{\prime}}{b\left(0_{+}\right)}+B_{1} * L^{\prime}+B_{2} * L, \text { on }\left(-\infty, t_{0}\right)
$$

This is $w=\mathcal{S}_{t_{0}, q}(l)$. This proves the Theorem.

We now have the following result:

Proposition 3.1. There exists $n_{0} \in \mathbb{N}$ s.t. for any $n \in \mathbb{N}, n \geq n_{0}$, the function $a_{n}$ introduced in the string of assumptions $\left(a_{2}\right)-\left(a_{5}\right)$ satisfies the hypotheses $\left(b_{1}\right)$ - $\left(b_{3}\right)$ of Theorem 3.1. Moreover, functions $B_{1}, B_{2}$ of equations (3.16)-(3.17), having b substituted by $a_{n}$, belong to a ball of radius independent of $n$ that is included into $L^{1}(\mathbb{R})$.

Proof. One has:

$$
a_{n}\left(0_{+}\right)=\frac{1}{\pi} \lim _{k \rightarrow+\infty} \int_{-k}^{k} \mathcal{F} b(\omega) \mathrm{d} \omega=\frac{1}{\pi} \lim _{k \rightarrow+\infty} \int_{-k}^{k} \operatorname{Re}[\mathcal{F} b(\omega)] \mathrm{d} \omega
$$

Invoking the hypothesis $\left(a_{4}\right)$ leads to

$$
a_{n}\left(0_{+}\right) \geq M_{1}
$$

meaning $a_{n}$ satisfies $\left(b_{1}\right)$. From $\left(a_{5}\right)$ we see that $a_{n}$ satisfies $\left(b_{3}\right)$.

Moreover, one has $\left(\mathcal{F} e^{-t}\right) \omega=\frac{1}{1+\omega^{2}}$. This fact, together with Theorem 2.4 on page 494 of [24] imply that the function $t \in[0,+\infty) \rightarrow a_{n}(t)-M_{4} e^{-t}$ is of positive type. From the same Theorem one also gets $\operatorname{Re}\left(\mathcal{L}\left(a_{n}-M_{1} e^{-t}\right)(z)\right) \geq 0$, for any $z \in \mathbb{C}$ s.t. $\operatorname{Re}(z) \geq 0$ The later in turn implies $\operatorname{Re}\left(\mathcal{L} a_{n}(z)\right) \geq M_{1} \frac{1+z_{1}}{\left(1+z_{1}\right)^{2}+z_{2}^{2}}$, for any $z=z_{1}+i z_{2}, z_{1} \geq 0$. Then $a_{n}$ satisfies $\left(b_{2}\right)$ with $\beta=2$.

The last statement of the Proposition is obvious.

\section{Approximated problems and estimates.}

\subsection{Approximated and local problems. Preliminary notations and estimates.}

Remark that $a$ is not smooth enough to ensure a straightforward local in time existence result for a solution $v$ to our problem. As a consequence we study the following approximated problem which we denote by $P_{n}$.

Problem $\mathbf{P}_{\mathbf{n}}$ : find $v_{n}: \Omega \times[0,+\infty) \rightarrow \mathbb{R}$ s.t.

$\left(P_{n}\right)_{1}\left(v_{n}\right)_{t}=\int_{0}^{+\infty} a_{n}^{\prime}(s) \frac{\partial}{\partial x} g\left(\left(\bar{v}_{n}^{t}\right)_{x}\right)(x, s) \mathrm{d} s+f(x, t)$ 
$\left(P_{n}\right)_{2} \quad v_{n}=0$ on $\partial \Omega, v_{n}(t)=0, \forall t<0$

$\left(P_{n}\right)_{3} v_{n}(x, 0)=v_{0}(x)$ for $x \in \Omega$

Given the assumptions on $g$ we conclude there exist $\gamma>0$ and $\theta \in[0,1]$ s.t.

$$
g^{\prime}(y)<-\gamma, \forall y \in[-\theta, \theta]
$$

Clearly we can take the same $\theta$ as in assumption $\left(g_{1}\right)$. Moreover, there exists $K>0$ s.t.

$$
\left|g^{\prime}(y)-g^{\prime}(0)\right| \leq K y^{2}, \forall y \in[-\theta, \theta]
$$

In the above one may consider the same $K$ as in $\left(g_{1}\right)$.

Let us denote, for almost every $x \in \Omega$,

$$
u_{n}(x, t)=\int_{0}^{t} v_{n}(x, s) d s
$$

The proof of the next Proposition is very similar to that of Theorem III.10 in [37] and is omitted.

Proposition 4.1. Assume that the hypotheses $\left(g_{1}\right)-\left(g_{3}\right),\left(f_{1}\right)-\left(f_{4}\right),\left(v_{0}\right)$, and $\left(a_{1}\right)-\left(a_{5}\right)$ on the data hold true. Then the initial value problem $\left(P_{n}\right)_{1},\left(P_{n}\right)_{2},\left(P_{n}\right)_{3}$ has a unique solution $v_{n}$ defined on a maximal time interval $\left[0, T_{n}\right), T_{n}>0$, and s.t. $v_{n} \in \mathscr{C}^{0}\left(\left[0, T_{n}\right) ; H^{2}(\Omega)\right),\left(v_{n}\right)_{t} \in$ $\mathscr{C}^{0}\left(\left[0, T_{n}\right) ; H^{1}(\Omega)\right),\left(v_{n}\right)_{t t} \in \mathscr{C}^{0}\left(\left[0, T_{n}\right) ; L^{2}(\Omega)\right)$ and $u_{n} \in \mathscr{C}^{0}\left(\left[0, T_{n}\right) ; H^{3}(\Omega)\right)$. Moreover, if

$$
\begin{aligned}
& \sup _{t \in\left[0, T_{n}\right)}\left\{\left\|v_{n}(\cdot, t)\right\|_{H^{2}(\Omega)}^{2}+\left\|\left(v_{n}\right)_{t}(\cdot, t)\right\|_{H^{1}(\Omega)}^{2}+\left\|\left(v_{n}\right)_{t t}(\cdot, t)\right\|_{L^{2}(\Omega)}^{2}+\left\|u_{n}(\cdot, t)\right\|_{H^{3}(\Omega)}^{2}\right\}<\infty \\
& \text { and }
\end{aligned}
$$

$$
\sup _{\substack{x \in \Omega \\ 0 \leq t \leq T_{n}}}\left|\left(u_{n}\right)_{x}(x, t)\right| \leq \frac{\theta}{2}
$$

with $\theta$ as in $\left(g_{4}\right)$, then $T_{n}=+\infty$.

Notice that our functional framework is different from that of [8]. As a consequence, here it is necessary to obtain new estimates on $\left\|u_{n}\right\|_{H^{3}(\Omega)}$.

In this Section we obtain the necessary estimates to proving $T_{n}=+\infty$. These estimates will be proved to be independent of $n$, fact which allows to pass to the limit as $n \rightarrow+\infty$. To simplify notations, we drop the subscript $n$ of $a_{n}, v_{n}$ and $T_{n}$.

Drawing inspiration from [8], we introduce the following expressions:

$$
\begin{aligned}
\mathcal{E}(t) & =\sup _{s \in[0, t)}\left[\int_{\Omega}\left(v^{2}+v_{x}^{2}+v_{t}^{2}+v_{x x}^{2}+v_{x t}^{2}+v_{t t}^{2}+u^{2}+u_{x}^{2}+u_{x x}^{2}+u_{x x x}^{2}\right)(x, s) \mathrm{d} x\right] \\
& +\int_{0}^{t} \int_{\Omega}\left(v^{2}+v_{x}^{2}+v_{t}^{2}+v_{x x}^{2}+v_{x t}^{2}+v_{t t}^{2}\right)(x, s) \mathrm{d} x \mathrm{~d} s \\
& =\sup _{s \in[0, t)}\left(\|v\|_{H^{2}(\Omega)}^{2}+\left\|v_{t}\right\|_{H^{1}(\Omega)}^{2}+\left\|v_{t t}\right\|_{L^{2}(\Omega)}^{2}+\|u\|_{H^{3}(\Omega)}^{2}\right)(s)+\|v\|_{H^{2}(\Omega \times[0, t))}^{2}
\end{aligned}
$$

and

$$
\nu(t)=\sup _{\substack{x \in \Omega \\ s \in[0, t}}\left[\sqrt{\left(v^{2}+v_{x}^{2}+v_{t}^{2}\right)(x, s)}\right]+\sqrt{\int_{0}^{t} \sup _{x \in \Omega}\left(v_{x}(x, s)\right)^{2} \mathrm{~d} s}
$$


For simplicity let us denote

$$
\begin{aligned}
\mathcal{E}_{1}(t)= & \sup _{s \in[0, t)}\left[\int_{\Omega}\left(v^{2}+v_{x}^{2}+v_{t}^{2}+v_{x t}^{2}+v_{t t}^{2}\right)(x, s) \mathrm{d} x\right]+ \\
& \int_{0}^{t} \int_{\Omega}\left(v^{2}+v_{x}^{2}+v_{t}^{2}+v_{x t}^{2}\right)(x, s) \mathrm{d} x \mathrm{~d} s
\end{aligned}
$$

In fact $\mathcal{E}_{1}(t)$ collects the terms of $\mathcal{E}(t)$ which will be estimated in a first step with the help of energy estimates.

Remark that, due to Sobolev inequalities, there exists a constant $C_{\Omega}>0$ s.t.

$$
\nu(t) \leq C_{\Omega} \sqrt{\mathcal{E}(t)}, \forall t \in[0, T)
$$

and

$$
\sup _{x \in \Omega}\left|u_{x}(x, t)\right| \leq C_{\Omega} \sqrt{\mathcal{E}(t)}, \forall t \in[0, T)
$$

Next, from (2.6) we get

$$
\begin{aligned}
\mathcal{G}_{t}(x, t) & =v_{x x}(x, t) \int_{0}^{+\infty} a^{\prime}(s)\left[g^{\prime}\left(\bar{v}_{x}^{t}(x, s)\right)-g^{\prime}(0)\right] \mathrm{d} s \\
& -\int_{0}^{t} v_{x x}(x, s) a^{\prime}(t-s)\left[g^{\prime}\left(\bar{v}_{x}^{t}(x, t-s)\right)-g^{\prime}(0)\right] \mathrm{d} s \\
& +\int_{0}^{t} v_{x x}(x, s) \int_{t-s}^{+\infty} a^{\prime}(\tau) g^{\prime \prime}\left(\bar{v}_{x}^{t}(x, \tau)\right)\left[v_{x}(x, t)-v_{x}(x, t-\tau)\right] \mathrm{d} \tau \mathrm{d} s
\end{aligned}
$$

All subsequent estimates will be obtained under the following smallness hypothesis on $\mathcal{E}(t)$ :

$$
\mathcal{E}(t) \leq \frac{\theta^{2}}{4 C_{\Omega}^{2}}, \forall t \in[0, T)
$$

which implies

$$
\sup _{\substack{x \in \Omega \\ 0 \leq t \leq T}}\left|u_{x}(x, t)\right| \leq \frac{\theta}{2}
$$

Then

$$
\sup _{\substack{x \in \Omega \\ 0 \leq s \leq t \leq T}}\left|\bar{v}_{x}^{t}(x, s)\right| \leq \theta, \text { a.e. } x \in \Omega
$$

We shall frequently employ the following inequalities:

$$
|x y| \leq \mu x^{2}+\frac{1}{4 \mu} y^{2}, x, y \in \mathbb{R}, \mu>0
$$

and

$$
\left\|F_{1} * F_{2}\right\|_{L^{p}(0, T)} \leq\left\|F_{1}\right\|_{L^{1}(0,+\infty)}\left\|F_{2}\right\|_{L^{p}(0, T)}
$$

for any $T>0, F_{1} \in L^{1}(0,+\infty)$, and $F_{2} \in L^{p}(0, T)$, with $p \geq 1$; f unctions $F_{1}$ and $F_{2}$ are extended to $\mathbb{R}$ by 0 .

For future reference we prove the following Lemmas: 
Lemma 4.1. Let the mappings $\varphi$ and $s \mapsto s \varphi(s)$ be elements of $L^{1}\left(\mathbb{R}_{+}\right)$. Then the function $s \mapsto \int_{s}^{+\infty} \varphi(\tau) \mathrm{d} \tau$ belongs to $L^{1}\left(\mathbb{R}_{+}\right)$and we have the estimate

$$
\int_{0}^{+\infty}\left|\int_{s}^{+\infty} \varphi(\tau) \mathrm{d} \tau\right| \mathrm{d} s \leq \int_{0}^{+\infty}|s \varphi(s)| \mathrm{d} s
$$

Proof. The proof is a direct consequence of Fubini's Theorem.

Lemma 4.2. Let $\varphi \in L^{1}\left(\mathbb{R}_{+}\right)$. Then:

(i) for any $w_{1}, w_{2} \in L^{2}\left(Q_{t}\right)$ we have

$$
\left|\int_{0}^{t} \int_{\Omega} w_{1}(x, s)\left(w_{2} * \varphi\right)(x, s) \mathrm{d} s\right| \leq\|\varphi\|_{L^{1}\left(\mathbb{R}_{+}\right)}\left\|w_{1}\right\|_{L^{2}\left(Q_{t}\right)}\left\|w_{2}\right\|_{L^{2}\left(Q_{t}\right)}
$$

(ii) for any $w_{3} \in L^{2}(\Omega), w_{4} \in L^{\infty}\left(0, T ; L^{2}(\Omega)\right)$ we have

$$
\left|\int_{\Omega} w_{3}(x)\left(\varphi * w_{4}\right)(x, t) \mathrm{d} x\right| \leq\|\varphi\|_{L^{1}(0, T)}\left\|w_{3}\right\|_{L^{2}(\Omega)} \sup _{0 \leq \tau \leq t}\left\|w_{4}(\tau)\right\|_{L^{2}(\Omega)}, \text { a.e.t } \in[0, T)
$$

Proof. Part (i): observe that

$$
\begin{aligned}
\left|\int_{0}^{t} \int_{\Omega} w_{1}(x, s)\left(w_{2} * \varphi\right)(x, s) \mathrm{d} s\right| & \leq \int_{\Omega}\left\|w_{1}(x, \cdot)\right\|_{L^{2}(0, t)}\left\|\left(w_{2} * \varphi\right)(x, \cdot)\right\|_{L^{2}(0, t)} \mathrm{d} x \\
& \leq\|\varphi\|_{L^{1}\left(\mathbb{R}_{+}\right)} \int_{\Omega}\left\|w_{1}(x, \cdot)\right\|_{L^{2}(0, t)}\left\|w_{2}(x, \cdot)\right\|_{L^{2}(0, t)} \mathrm{d} x
\end{aligned}
$$

which gives the result.

Part (ii): one has

$$
\left|\int_{\Omega} w_{3}(x)\left(\varphi * w_{4}\right)(x, t) \mathrm{d} x\right| \leq\left\|w_{3}\right\|_{L^{2}(\Omega)} \int_{0}^{t}\left\|w_{4}(x, t-\tau)\right\|_{L^{2}(\Omega)}|\varphi(\tau)| \mathrm{d} \tau
$$

and the result follows.

Let $r_{0}: \mathbb{R}_{+} \rightarrow \mathbb{R}_{+}, r_{0}(s):=\min \{s, \sqrt{s}\}$. We have the following estimates:

Lemma 4.3. Let $t \in[0, T)$, assume 4.10 is satisfied. Then:

(i) $\left|g^{(j)}\left(\bar{v}_{x}^{t}(x, s)\right)-g^{(j)}(0)\right| \leq K \min \left\{\nu(t) r_{0}(s), \theta\right\}$ a.e. $x \in \Omega, s \in[0, t], j=0,1,2,3$

(ii) $|\mathcal{G}(x, t)| \leq K \nu(t)\left[\left|v_{x x}(x, \cdot)\right| * \psi\right](t)$, a.e. $x \in \Omega$

(iii) $\left|\mathcal{G}_{t}(x, t)\right| \leq K \nu(t) \bar{a}\left|v_{x x}(x, t)\right|+K \nu(t)\left[\left|v_{x x}(x, \cdot)\right| * \psi\right](t)$, a.e. $x \in \Omega$,

where

$$
\begin{gathered}
\bar{a}=\int_{0}^{+\infty}\left|a^{\prime}(s)\right| r_{0}(s) \mathrm{d} s \\
\psi(t)=\left|a^{\prime}(t)\right| r_{0}(t)+2 \int_{t}^{+\infty}\left|a^{\prime}(\tau)\right| r_{0}(\tau) \mathrm{d} \tau
\end{gathered}
$$


Remark 4.1. Lemma 4.1 and the assumptions made about function a grant the fact that $\psi$ in (4.20) is s.t. $\psi \in L^{1}\left(\mathbb{R}_{+}\right)$.

Proof. (i) On one hand, as a consequence of $\left(g_{1}\right)$ and 4.12 we have

$$
\left|g^{(j)}\left(\bar{v}_{x}^{t}(x, s)\right)-g^{(j)}(0)\right| \leq K\left|\bar{v}_{x}^{t}(x, s)\right|, j=0,1,2,3
$$

On the other hand,

$$
\left|\bar{v}_{x}^{t}(x, s)\right| \leq \int_{t-s}^{t}\left|v_{x}(x, \lambda)\right| \mathrm{d} \lambda \leq s \sup _{t-s \leq \lambda \leq t}\left|v_{x}(x, \lambda)\right| \leq s \nu(t)
$$

and

$$
\left|\bar{v}_{x}^{t}(x, s)\right| \leq \sqrt{s}\left[\int_{t-s}^{t}\left|v_{x}(x, \lambda)\right|^{2} \mathrm{~d} \lambda\right]^{1 / 2} \leq \sqrt{s} \nu(t)
$$

which gives the result.

(ii) From (2.6) and (ii) above one gets:

$$
\begin{aligned}
|\mathcal{G}(x, t)| & \leq K \nu(t) \int_{0}^{t}\left|v_{x x}(x, s)\right| \int_{t-s}^{+\infty} a^{\prime}(\tau) \min \{\tau, \sqrt{\tau}\} \mathrm{d} \tau \mathrm{d} s \\
& \leq K \nu(t) \int_{0}^{t}\left|v_{x x}(x, s)\right| \psi(t-s) \mathrm{d} s
\end{aligned}
$$

from which the result follows.

(iii) We use (4.9), $\left(g_{1}\right)$, (ii), the fact that $g^{\prime \prime}(0)=0$ and $0 \leq \theta \leq 1$ to obtain:

$$
\begin{aligned}
\left|\mathcal{G}_{t}(x, t)\right| & \leq K\left|v_{x x}(x, t)\right| \nu(t) \int_{0}^{+\infty}\left|a^{\prime}(s)\right| r_{0}(s) \mathrm{d} s \\
& +K \nu(t) \int_{0}^{t}\left|v_{x x}(x, s)\right|\left|a^{\prime}(t-s)\right| r_{0}(t-s) \mathrm{d} s \\
& +2 K \theta \nu(t) \int_{0}^{t}\left|v_{x x}(x, s)\right| \int_{t-s}^{+\infty}\left|a^{\prime}(\tau)\right| r_{0}(\tau) \mathrm{d} \tau \mathrm{d} s
\end{aligned}
$$

which gives the result.

\subsection{Energy estimates.}

The next Lemmas give energy estimates for the terms in $\mathcal{E}_{1}(t)$ (see (4.6)), as in [8].

In what follows, the notation $C>0$ stands for a generic constant that is independent of $n$.

Lemma 4.4. Assume the inequality 4.10 holds true. Then

$$
\int_{\Omega} v^{2}(x, t) \mathrm{d} x-2 g^{\prime}(0) Q\left(v_{x}, t, a\right) \leq V_{0}+2 \sqrt{F} \sqrt{\mathcal{E}(t)}+2 K\|\psi\|_{L^{1}\left(\mathbb{R}_{+}\right)} \nu(t) \mathcal{E}(t)
$$


Proof. For a fixed $t \in\left(0, T_{0}\right)$, we multiply $(2.5)$ by $v(x, t)$ and integrate on $\Omega$ and on $(0, t)$. We get

$$
\begin{aligned}
& \frac{1}{2} \int_{\Omega} v^{2}(x, t) \mathrm{d} x-\frac{1}{2} \int_{\Omega} v_{0}^{2} \mathrm{~d} x-g^{\prime}(0) Q\left(v_{x}, t, a\right) \\
& =\int_{0}^{t} \int_{\Omega} f(x, s) v(x, s) \mathrm{d} x \mathrm{~d} s+\int_{0}^{t} \int_{\Omega} \mathcal{G}(x, s) v(x, s) \mathrm{d} x \mathrm{~d} s
\end{aligned}
$$

Observe that $\int_{0}^{t} \int_{\Omega} f v \mathrm{~d} x \mathrm{~d} s \leq\|f\|_{L^{2}\left(Q_{t}\right)}\|v\|_{L^{2}\left(Q_{t}\right)} \leq \sqrt{F} \sqrt{\mathcal{E}}$.

Now, using Lemma 4.3 we get

$$
\left|\int_{0}^{t} \int_{\Omega} \mathcal{G}(x, s) v(x, s) \mathrm{d} x \mathrm{~d} s\right| \leq K \nu(t) \int_{0}^{t} \int_{\Omega}|v(x, s)|\left(\left|v_{x x}\right| *|\psi|\right)(x, s) \mathrm{d} x \mathrm{~d} s
$$

Using part (i) of Lemma 4.2 with $w_{1}=v, w_{2}=v_{x x}$ and $\varphi=|\psi|$ one gets

$$
\left|\int_{0}^{t} \int_{\Omega} \mathcal{G}(x, s) v(x, s) \mathrm{d} x \mathrm{~d} s\right| \leq K \nu(t)\|\psi\|_{L^{1}\left(\mathbb{R}_{+}\right)} \mathcal{E}(t)
$$

thus ending the proof.

Lemma 4.5. Let $\bar{a}$ and $\psi$ be given by (4.19) and (4.20), respectively. Under the assumption that 4.10) is fulfilled, one has the following inequality:

$$
\begin{aligned}
\int_{\Omega} v_{t}^{2}(x, t) \mathrm{d} x-2 g^{\prime}(0) Q\left(v_{x t}, t, a\right) & \leq F+2\|a\|_{L^{1}\left(\mathbb{R}_{+}\right)} \sqrt{V_{0}} \sqrt{\mathcal{E}(t)} \\
& +2 \sqrt{F} \sqrt{\mathcal{E}(t)}+2 K\left(\|\psi\|_{L^{1}\left(\mathbb{R}_{+}\right)}+\bar{a}\right) \nu(t) \mathcal{E}(t)
\end{aligned}
$$

Proof. First, we derivate 2.5 w.r.t. $t$ and obtain

$$
v_{t t}(x, t)+g^{\prime}(0) a(0) v_{x x}(x, t)+g^{\prime}(0) \int_{0}^{t} a^{\prime}(t-s) v_{x x}(x, s) \mathrm{d} s=f_{t}+\mathcal{G}_{t}
$$

Secondly, multiplying the above by $v_{t}$ and integrating on $\Omega$ and on $[0, t]$ leads to

$$
\begin{aligned}
& \frac{1}{2} \int_{\Omega} v_{t}^{2}(x, t) \mathrm{d} x-\frac{1}{2} \int_{\Omega} v_{t}^{2}(x, 0) \mathrm{d} x-g^{\prime}(0) a(0) \int_{0}^{t} \int_{\Omega} v_{x} v_{x t} \mathrm{~d} x \mathrm{~d} s \\
& -g^{\prime}(0) \int_{0}^{t} \int_{\Omega} \int_{0}^{s} a^{\prime}(s-\tau) v_{x}(\tau) \mathrm{d} \tau v_{x t}(s) \mathrm{d} x \mathrm{~d} s=\int_{0}^{t} \int_{\Omega} f_{t} v_{t} \mathrm{~d} x \mathrm{~d} s+\int_{0}^{t} \int_{\Omega} \mathcal{G}_{t} v_{t} \mathrm{~d} x \mathrm{~d} s
\end{aligned}
$$

Observe now that

$$
\int_{0}^{s} a^{\prime}(s-\tau) v_{x}(\tau) \mathrm{d} \tau=-a(0) v_{x}(s)+a(s) v_{x}(0)+\int_{0}^{s} a(s-\tau) v_{x t}(\tau) \mathrm{d} \tau
$$

One now gets:

$$
\begin{aligned}
& \frac{1}{2} \int_{\Omega} v_{t}^{2}(x, t) \mathrm{d} x-g^{\prime}(0) Q\left(v_{x t}, t, a\right)=\frac{1}{2} \int_{\Omega} v_{t}^{2}(x, 0) \mathrm{d} x-g^{\prime}(0) \int_{0}^{t} \int_{\Omega} a(s) v_{0}^{\prime \prime}(x) v_{t}(x, s) \mathrm{d} x \mathrm{~d} s \\
& +\int_{0}^{t} \int_{\Omega}\left(f_{t} v_{t}\right)(x, s) \mathrm{d} x \mathrm{~d} s+\int_{0}^{t} \int_{\Omega}\left(\mathcal{G}_{t} v_{t}\right)(x, s) \mathrm{d} x \mathrm{~d} s
\end{aligned}
$$


Notice that

$$
v_{t}(x, 0)=f(x, 0)
$$

which gives $\int_{\Omega} v_{t}^{2}(x, 0) \mathrm{d} x \leq F$. We also have

$$
\begin{aligned}
\left|\int_{0}^{t} \int_{\Omega} a(s) v_{0}^{\prime \prime}(x) v_{t}(x, s) \mathrm{d} x \mathrm{~d} s\right| & \leq\left\|v_{0}^{\prime \prime}\right\|_{L^{2}(\Omega)}\|a\|_{L^{1}\left(\mathbb{R}_{+}\right)} \sup _{0 \leq s \leq t}\left\|v_{t}(\cdot, s)\right\|_{L^{2}(\Omega)} \\
& \leq\|a\|_{L^{1}\left(\mathbb{R}_{+}\right)} \sqrt{V_{0}} \sqrt{\mathcal{E}(t)}
\end{aligned}
$$

and

$$
\int_{0}^{t} \int_{\Omega}\left(f_{t} v_{t}\right)(x, s) \mathrm{d} x \mathrm{~d} s \leq \sqrt{F} \sqrt{\mathcal{E}(t)}
$$

Finally, invoking part (iii) of Lemma 4.3 and part (i) of Lemma 4.2 we deduce that

$$
\int_{0}^{t} \int_{\Omega}\left(\mathcal{G}_{t} v_{t}\right)(x, s) \mathrm{d} x \mathrm{~d} s \leq K \bar{a} \nu(t) \mathcal{E}(t)+K \nu(t)\|\psi\|_{L^{1}\left(\mathbb{R}_{+}\right)} \mathcal{E}(t)
$$

and with the obtainment of this last estimates the proof ends.

Next, in order to obtain energy estimates for $\int_{\Omega} v_{t t}^{2}(x, t) \mathrm{d} x$ we shall use the difference operator $\left(\triangle_{h} w\right)(x, t)=w(x, t+h)-w(x, t)$, for $h>0$ small enough.

Lemma 4.6. Under the assumption that 4.10 is fulfilled, one has:

$$
\begin{aligned}
\int_{\Omega} v_{t t}^{2}(x, t) \mathrm{d} x-2 g^{\prime}(0) \lim _{h \rightarrow 0_{+}} \frac{1}{h^{2}} Q\left(\triangle_{h} v_{x t}, t, a\right) \leq & C\{F+\sqrt{F} \sqrt{\mathcal{E}(t)} \\
& \left.+\left[\nu(t)+\nu^{3}(t)\right] \mathcal{E}(t)+\sqrt{V_{0}} \mathcal{E}(t)\right\}
\end{aligned}
$$

For the Proof, see the Appendix Section.

Since $\nu(t)$ and $\mathcal{E}(t)$ are non-increasing functions in $t$, we obtain as a consequence of Lemma 4.4. Lemma 4.5, Lemma 4.6, Lemma 3.1 and Sobolev embeddings, that:

Lemma 4.7. Under the assumption stated in 4.10 one has

$$
\mathcal{E}_{1}(t) \leq C\left\{V_{0}+F+\left(\sqrt{V_{0}}+\sqrt{F}\right) \sqrt{\mathcal{E}(t)}+\left[\nu(t)+\nu^{3}(t)\right] \mathcal{E}(t)+\sqrt{V_{0}} \mathcal{E}(t)\right\}
$$

\subsection{Non-energy estimates.}

In the following we obtain estimates for the other constitutive terms of $\mathcal{E}(t)$.

Now, from (2.5) and using for a.e. $x \in \Omega$ the result of Theorem 3.1 with $b=a$ (see Proposition 3.1,

$$
\begin{gathered}
l(t)=\frac{1}{g^{\prime}(0)}\left[f(x, t)+\mathcal{G}(x, t)-v_{t}(x, t)\right], \text { and } w(t)=v_{x x}(x, t), \text { we deduce the equality } \\
v_{x x}=\frac{1}{g^{\prime}(0)}\left[\frac{1}{a(0)}\left(f_{t}+\mathcal{G}_{t}-v_{t t}\right)+A_{1} *\left(f_{t}+\mathcal{G}_{t}-v_{t t}\right)+A_{2} *\left(f+\mathcal{G}-v_{t}\right)\right]
\end{gathered}
$$

where $A_{1}, A_{2} \in L_{[0,+\infty)}^{1}(\mathbb{R})$ are two functions that depend on $a_{n}$, with bounded $L^{1}$ norms which are independent of $n$, due to Proposition 3.1.

We have the following estimate: 
Lemma 4.8. Under the assumption stated in (4.10) one has

$$
\begin{aligned}
& \int_{\Omega} v_{x x}^{2}(x, t) \mathrm{d} x+\int_{0}^{t} \int_{\Omega} v_{x x}^{2}(x, s) \mathrm{d} x \mathrm{~d} s+\int_{0}^{t} \int_{\Omega} v_{t t}^{2}(x, s) \mathrm{d} x \mathrm{~d} s \\
& \leq C\left[F+\mathcal{E}_{1}(t)+\nu(t) \mathcal{E}(t)\right]
\end{aligned}
$$

\section{Proof. Step 1.}

We multiply (4.39) by $v_{x x}$ and integrate on $\Omega$. It is clear that, for any $\eta>0$, we have

$$
\left|\int_{\Omega}\left(f_{t}-v_{t t}\right) v_{x x} \mathrm{~d} x\right| \leq \eta \int_{\Omega} v_{x x}^{2} \mathrm{~d} x+\frac{1}{2 \eta} \int_{\Omega}\left(f_{t}^{2}+v_{t t}^{2}\right) \mathrm{d} x
$$

From part (iii) in Lemma 4.3 we obtain

$$
\begin{aligned}
\left|\int_{\Omega} \mathcal{G}_{t} v_{x x} \mathrm{~d} x\right| & \leq K \nu(t) \int_{\Omega}\left|v_{x x}(x, t)\right|\left(\left|v_{x x}\right| *|\psi|\right)(x, t) \mathrm{d} x \\
& +\bar{a} K \nu(t) \int_{\Omega}\left|v_{x x}(x, t)\right|^{2} \mathrm{~d} x
\end{aligned}
$$

Further, with the help of part (ii) in Lemma 4.2 we obtain

$$
\begin{aligned}
\left|\int_{\Omega} \mathcal{G}_{t} v_{x x} \mathrm{~d} x\right| & \leq K \nu(t)\left\|v_{x x}(\cdot, t)\right\|_{L^{2}(\Omega)}\|\psi\|_{L^{1}\left(\mathbb{R}_{+}\right)} \sup _{0 \leq \tau \leq t}\left\|v_{x x}(\cdot, \tau)\right\|_{L^{2}(\Omega)} \\
& +\bar{a} K \nu(t)\left\|v_{x x}(\cdot, t)\right\|_{L^{2}(\Omega)}^{2} \leq K \nu(t)\left[\|\psi\|_{L^{1}\left(\mathbb{R}_{+}\right)}+\bar{a}\right] \mathcal{E}(t)
\end{aligned}
$$

For any $\eta>0$ one has

$$
\begin{aligned}
& \left|\int_{\Omega} A_{1} *\left(f_{t}-v_{t t}\right) v_{x x} \mathrm{~d} x\right| \leq\left\|A_{1}\right\|_{L^{1}\left(\mathbb{R}_{+}\right)}\left\|v_{x x}(\cdot, t)\right\|_{L^{2}(\Omega)} \sup _{0 \leq \tau \leq t}\left[\left\|f_{t}(\cdot, \tau)\right\|_{L^{2}(\Omega)}+\left\|v_{t t}(\cdot, \tau)\right\|_{L^{2}(\Omega)}\right] \\
& \leq \eta\left\|v_{x x}(\cdot, t)\right\|_{L^{2}(\Omega)}^{2}+\frac{1}{2 \eta}\left\|A_{1}\right\|_{L^{1}\left(\mathbb{R}_{+}\right)}^{2} \sup _{0 \leq \tau \leq t}\left[\left\|f_{t}(\cdot, \tau)\right\|_{L^{2}(\Omega)}^{2}+\left\|v_{t t}(\cdot, \tau)\right\|_{L^{2}(\Omega)}^{2}\right]
\end{aligned}
$$

and also

$$
\begin{aligned}
& \left|\int_{\Omega} A_{2} *\left(f-v_{t}\right) v_{x x} \mathrm{~d} x\right| \\
& \leq \eta\left\|v_{x x}(\cdot, t)\right\|_{L^{2}(\Omega)}^{2}+\frac{1}{2 \eta}\left\|A_{2}\right\|_{L^{1}\left(\mathbb{R}_{+}\right)}^{2} \sup _{0 \leq \tau \leq t}\left[\|f(\cdot, \tau)\|_{L^{2}(\Omega)}^{2}+\left\|v_{t}(\cdot, \tau)\right\|_{L^{2}(\Omega)}^{2}\right]
\end{aligned}
$$

We now have:

$$
\begin{aligned}
\left|\int_{\Omega}\left(A_{1} * \mathcal{G}_{t}\right)(x, t) v_{x x}(x, t) \mathrm{d} x\right| & \leq \bar{a} K \nu(t) \int_{\Omega}\left(\left|A_{1}\right| *\left|v_{x x}\right|\right)(x, t)\left|v_{x x}(x, t)\right| \mathrm{d} x \\
& +K \nu(t) \int_{\Omega}\left(\left|A_{1}\right| *|\psi| *\left|v_{x x}(x, t)\right|\right)(x, t)\left|v_{x x}(x, t)\right| \mathrm{d} x
\end{aligned}
$$

Then: 


$$
\begin{aligned}
& \left|\int_{\Omega}\left(A_{1} * \mathcal{G}_{t}\right)(x, t) v_{x x}(x, t) \mathrm{d} x\right| \\
& \leq K \nu(t)\left[\bar{a}\left\|A_{1}\right\|_{L^{1}\left(\mathbb{R}_{+}\right)}+\left\|\left|A_{1}\right| *|\psi|\right\|_{L^{1}\left(\mathbb{R}_{+}\right)}\right]\left\|v_{x x}(\cdot, t)\right\|_{L^{2}(\Omega)} \sup _{0 \leq \tau \leq t}\left\|v_{x x}(\cdot, \tau)\right\|_{L^{2}(\Omega)} .
\end{aligned}
$$

This gives

$$
\left|\int_{\Omega}\left(A_{1} * \mathcal{G}_{t}\right)(x, t) v_{x x}(x, t) \mathrm{d} x\right| \leq C \nu(t) \mathcal{E}(t)
$$

Likewise,

$$
\left|\int_{\Omega}\left(A_{2} * \mathcal{G}\right)(x, t) v_{x x}(x, t) \mathrm{d} x\right| \leq C \nu(t) \mathcal{E}(t)
$$

Now, from the above estimates (4.41), 4.43), 4.44), 4.45), 4.48) and (4.49), with $\eta>0$ small enough leads to

$$
\sup _{0 \leq s \leq t} \int_{\Omega} v_{x x}^{2}(x, s) \mathrm{d} x \leq C\left[F+\mathcal{E}_{1}(t)+\nu(t) \mathcal{E}(t)\right]
$$

\section{Step 2.}

We multiply 4.39 by $v_{x x}$ and integrate on $(0, t)$ and on $\Omega$. Proceeding as in Step 1., using part (i) in Lemma 4.2 , one gets for any $\eta>0$ that

$$
\begin{aligned}
& \int_{Q_{t}}\left[f_{t}+\mathcal{G}_{t}+A_{1} * f_{t}+A_{2} *\left(f-v_{t}\right)+A_{1} * \mathcal{G}_{t}+A_{2} * \mathcal{G}\right] v_{x x} \mathrm{~d} x \mathrm{~d} s \\
& \leq \eta \int_{Q_{t}} v_{x x}^{2} \mathrm{~d} x \mathrm{~d} s+\frac{C}{\eta}\left[F+\mathcal{E}_{1}(t)\right]+C \nu(t) \mathcal{E}(t)
\end{aligned}
$$

We are left to focus on terms that contain $v_{t t}$. Invoking density arguments,

$$
\int_{Q_{t}}\left(v_{t t} v_{x x}\right)(x, s) \mathrm{d} x \mathrm{~d} s=\int_{\Omega}\left(v_{x x} v_{t}\right)(x, t) \mathrm{d} x-\int_{\Omega} v_{0}^{\prime \prime}(x) v_{t}(x, 0) \mathrm{d} x+\int_{Q_{t}} v_{x t}^{2} \mathrm{~d} x \mathrm{~d} s
$$

which gives, using 4.33),

$$
\begin{aligned}
\left|\int_{Q_{t}}\left(v_{t t} v_{x x}\right)(x, s) \mathrm{d} x \mathrm{~d} s\right| & \leq\left\|v_{x x}(\cdot, t)\right\|_{L^{2}(\Omega)}\left\|v_{t}(\cdot, t)\right\|_{L^{2}(\Omega)} \\
& +\left\|v_{0}^{\prime \prime}\right\|_{L^{2}(\Omega)}\|f(\cdot, 0)\|_{L^{2}(\Omega)}+\int_{Q_{t}} v_{x t}^{2}(x, s) \mathrm{d} x \mathrm{~d} s
\end{aligned}
$$

Finally we have:

$$
\begin{aligned}
\int_{Q_{t}}\left(A_{1} * v_{t t}\right)(x, s) v_{x x}(x, s) \mathrm{d} x \mathrm{~d} s & =\int_{Q_{t}}\left(A_{1} * v_{t}\right)_{t} v_{x x}(x, s) \mathrm{d} x \mathrm{~d} s \\
& -\int_{Q_{t}} A_{1}(s) v_{t}(x, 0) v_{x x}(x, s) \mathrm{d} x \mathrm{~d} s
\end{aligned}
$$

Again, calling in the density arguments leads to 


$$
\begin{aligned}
\int_{Q_{t}}\left(A_{1} * v_{t}\right)_{t}(x, s) v_{x x}(x, s) \mathrm{d} x \mathrm{~d} s & =\int_{\Omega}\left(A_{1} * v_{t}\right)(x, t) v_{x x}(x, t) \mathrm{d} x \\
& +\int_{Q_{t}}\left(A_{1} * v_{x t}\right) v_{x t} \mathrm{~d} x \mathrm{~d} s
\end{aligned}
$$

From equalities (4.54) and 4.55) one easily gets:

$$
\begin{aligned}
& \left|\int_{Q_{t}}\left(A_{1} * v_{t t}\right) v_{x x}(x, s) \mathrm{d} x \mathrm{~d} s\right| \leq\left\|A_{1}\right\|_{L^{1}\left(\mathbb{R}_{+}\right)} \\
& {\left[\int_{Q_{t}} v_{x t}^{2} \mathrm{~d} x \mathrm{~d} s+\left\|v_{x x}(\cdot, t)\right\|_{L^{2}(\Omega)} \sup _{0 \leq \tau \leq t}\left\|v_{t}(\cdot, t)\right\|_{L^{2}(\Omega)}+\|f(\cdot, 0)\|_{L^{2}(\Omega)} \sup _{0 \leq s \leq t}\left\|v_{x x}(\cdot, s)\right\|_{L^{2}(\Omega)}\right]}
\end{aligned}
$$

Now, adding inequalities 4.51), 4.53), 4.56) and upon using 4.50) it allows us to get

$$
\int_{Q_{t}} v_{x x}^{2}(x, t) \mathrm{d} x \leq C\left[F+\mathcal{E}_{1}(t)+\nu(t) \mathcal{E}(t)\right]
$$

\section{Step 3.}

We now multiply $(4.29)$ by $v_{t t}$ and integrate on $Q_{t}$. We have the listed below results:

$$
\begin{gathered}
\left|\int_{Q_{t}} v_{x x} v_{t t} \mathrm{~d} x \mathrm{~d} s\right| \leq \eta \int_{Q_{t}} v_{t t}^{2} \mathrm{~d} x \mathrm{~d} s+\frac{1}{4 \eta} \int_{Q_{t}} v_{x x}^{2} \mathrm{~d} x \mathrm{~d} s \\
\int_{Q_{t}}\left(a^{\prime} * v_{x x}\right) v_{t t} \mathrm{~d} x \mathrm{~d} s \leq\left\|a^{\prime}\right\|_{L^{1}\left(\mathbb{R}_{+}\right)}\left\|v_{x x}\right\|_{L^{2}\left(Q_{t}\right)}\left\|v_{t t}\right\|_{L^{2}\left(Q_{t}\right)} \\
\leq \eta\left\|v_{t t}\right\|_{L^{2}\left(Q_{t}\right)}^{2}+\frac{1}{4 \eta}\left\|a^{\prime}\right\|_{L^{1}\left(\mathbb{R}_{+}\right)}^{2}\left\|v_{x x}\right\|_{L^{2}\left(Q_{t}\right)}^{2} \\
\int_{Q_{t}} f_{t} v_{t t} \mathrm{~d} x \mathrm{~d} s \leq \eta\left\|v_{t t}\right\|_{L^{2}\left(Q_{t}\right)}^{2}+\frac{1}{4 \eta}\left\|f_{t}\right\|_{L^{2}\left(Q_{t}\right)}^{2} \\
\int_{Q_{t}} \mathcal{G}_{t} v_{t t} \mathrm{~d} x \mathrm{~d} s \leq \bar{a} k \nu(t) \int_{Q_{t}}\left|v_{x x}\right|\left|v_{t t}\right| \mathrm{d} x \mathrm{~d} s+k \nu(t) \int_{Q_{t}}\left(\left|v_{x x}\right| *|\psi|\right)\left|v_{t t}\right| \mathrm{d} x \mathrm{~d} s \\
\leq k \nu(t)\left(\bar{a}+\|\psi\|_{L^{1}\left(\mathbb{R}_{+}\right)}\right) \mathcal{E}(t)
\end{gathered}
$$

We then obtain, taking $\eta$ small enough and using (4.57), that

$$
\int_{Q_{t}} v_{x x}^{2}(x, t) \mathrm{d} x \mathrm{~d} s \leq C\left[F+\mathcal{E}_{1}(t)+\nu(t) \mathcal{E}(t)\right]
$$

Now from estimates 4.50), 4.57) and 4.62) we obtain the result of Lemma 4.8.

Now we take on to obtaining estimates for $u$ defined as $u(x, t)=\int_{0}^{t} v(x, s) \mathrm{d} s$. The idea is to integrate 4.39 w.r.t. $t$; one gets:

$$
\begin{aligned}
& u_{x x}= \\
& \frac{1}{g^{\prime}(0)}\left\{\frac{f+\mathcal{G}-v_{t}}{a(0)}+\int_{0}^{t}\left[A_{1} *\left(f_{t}+\mathcal{G}_{t}-v_{t t}\right)\right](x, s) \mathrm{d} s+\int_{0}^{t}\left[A_{2} *\left(f+\mathcal{G}-v_{t}\right)\right](x, s) \mathrm{d} s\right\}
\end{aligned}
$$

We shall use in the following the below Lemma: 
Lemma 4.9. Suppose that $A \in L^{1}(0, T), \varphi \in W^{1,1}(0, T)$. Then, for any $t \in(0, T)$, we have

$$
\int_{0}^{t}\left(A * \varphi^{\prime}\right)(s) \mathrm{d} s=A *[\varphi-\varphi(0) H]
$$

Proof. The proof is a direct consequence of Fubini's Theorem.

Recall from 4.33) that $\left(f+\mathcal{G}-v_{t}\right)(x, 0)=0$. Then 4.63) can be re-written in the form

$$
\begin{aligned}
& u_{x x}= \\
& \frac{1}{g^{\prime}(0)}\left\{\frac{f+\mathcal{G}-v_{t}}{a(0)}+A_{1} *\left(f+\mathcal{G}-v_{t}\right)+A_{2} *\left[\int_{0}^{t} f(x, s) \mathrm{d} s+\int_{0}^{t} \mathcal{G}(x, s) \mathrm{d} s-v+v_{0}\right]\right\}
\end{aligned}
$$

We deduce from the above equation that

$$
\begin{aligned}
& u_{x x x}=\frac{1}{g^{\prime}(0)} \\
& \left\{\frac{f_{x}+\mathcal{G}_{x}-v_{x t}}{a(0)}+A_{1} *\left(f_{x}+\mathcal{G}_{x}-v_{x t}\right)+A_{2} *\left[\int_{0}^{t} f_{x}(x, s) \mathrm{d} s+\int_{0}^{t} \mathcal{G}_{x}(x, s) \mathrm{d} s-v_{x}+v_{0}^{\prime}\right]\right\}
\end{aligned}
$$

We can now prove the following:

Lemma 4.10. Consider the assumption formulated in 4.10 holds true. Then

$$
\sup _{0 \leq s \leq t}\left\|u_{x x}(\cdot, s)\right\|_{L^{2}(\Omega)}^{2} \leq C\left\{V_{0}+F+\nu^{2}(t) \mathcal{E}(t)+\mathcal{E}^{3}(t)+\mathcal{E}_{1}(t)\right\}
$$

and

$$
\sup _{0 \leq s \leq t}\left\|u_{x x x}(\cdot, s)\right\|_{L^{2}(\Omega)}^{2} \leq C\left\{V_{0}+F+\nu^{2}(t) \mathcal{E}(t)+\nu^{2}(t) \mathcal{E}^{2}(t)+\mathcal{E}^{3}(t)+\mathcal{E}_{1}(t)\right\}
$$

where $C>0$ is a constant which is independent of $n$.

Proof. The proof is performed in two steps.

\section{Step 1.}

Here we obtain the necessary estimates for $\mathcal{G}(t), \int_{0}^{t} \mathcal{G}(s) \mathrm{d} s, \mathcal{G}_{x}(t)$ and for $\int_{0}^{t} \mathcal{G}_{x}(s) \mathrm{d} s$. Using (2.6) and part (i) of Lemma 4.3 we have

$$
|\mathcal{G}(t)| \leq K \nu(t) \int_{0}^{+\infty}\left|a^{\prime}(s)\right| r_{0}(s)\left|u_{x x}(x, t)-u_{x x}(x, t-s)\right| \mathrm{d} s
$$

and this gives

$$
\|\mathcal{G}(\cdot, t)\|_{L^{2}(\Omega)} \leq 2 K \nu(t) \int_{0}^{+\infty}\left|a^{\prime}(s)\right| r_{0}(s) \mathrm{d} s\left(\sup _{0 \leq s \leq t}\left\|u_{x x}(\cdot, s)\right\|_{L^{2}(\Omega)}\right) \leq C \nu(t) \sqrt{\mathcal{E}(t)}
$$


On the other hand, using (2.6) and (4.2), we have that

$$
\left|\int_{0}^{t} \mathcal{G}(x, s) \mathrm{d} s\right| \leq K \int_{0}^{+\infty}\left|a^{\prime}(\tau)\right| \int_{0}^{t}\left|\bar{v}_{x}^{s}(x, \tau)\right|^{2}\left|u_{x x}(x, s)-u_{x x}(x, s-\tau)\right| \mathrm{d} s \mathrm{~d} \tau
$$

which implies, taking the $L^{2}(\Omega)$-norm, that

$$
\begin{aligned}
\left\|\int_{0}^{t} \mathcal{G}(\cdot, s) \mathrm{d} s\right\|_{L^{2}(\Omega)} \leq & 2 K\left(\sup _{0 \leq \tau \leq t}\left\|u_{x x}(\cdot, \tau)\right\|_{L^{2}(\Omega)}\right) \\
& \int_{0}^{+\infty}\left|a^{\prime}(\tau)\right| \int_{0}^{t}\left\|\bar{v}_{x}^{s}(\cdot, \tau)\right\|_{L^{\infty}(\Omega)}^{2} \mathrm{~d} s \mathrm{~d} \tau
\end{aligned}
$$

Now we have by Sobolev inclusions:

$$
\left\|\bar{v}_{x}^{s}(\cdot, \tau)\right\|_{L^{\infty}(\Omega)} \leq C \int_{s-\tau}^{s}\|v(\cdot, \lambda)\|_{H^{2}(\Omega)} \mathrm{d} \lambda \leq 2 C \tau \mathcal{M}\left(\|\tilde{v}\|_{H^{2}(\Omega)}\right)(s)
$$

where $\tilde{v}(x, s)$ is the function defined on $\Omega \times \mathbb{R}$ by

$$
\tilde{v}(x, s)= \begin{cases}v(x, s) & \text { for } s \in[0, t) \\ 0 & \text { for } s \in \mathbb{R}-[0, t)\end{cases}
$$

and

$$
\mathcal{M}\left(\|\tilde{v}\|_{H^{2}(\Omega)}\right)(s)=\sup _{\rho>0} \frac{1}{2 \rho} \int_{s-\rho}^{s+\rho}\|\tilde{v}(\cdot, \tau)\|_{H^{2}(\Omega)} \mathrm{d} \tau
$$

is the maximal function of $s \mapsto\|\tilde{v}(\cdot, s)\|_{H^{2}(\Omega)}$ (see [40]).

Now, the maximal inequality (see Theorem 1, page 5 in [40]) in this case leads to

$$
\begin{aligned}
\int_{\mathbb{R}} \mathcal{M}\left(\|\tilde{v}(\cdot, s)\|_{H^{2}(\Omega)}^{2}\right)(s) \mathrm{d} s & \leq 2 \sqrt{10} \int_{\mathbb{R}}\|\tilde{v}(\cdot, s)\|_{H^{2}(\Omega)}^{2}(x, s) \mathrm{d} s \\
& =2 \sqrt{10} \int_{0}^{t}\|v(\cdot, s)\|_{H^{2}(\Omega)}^{2}(x, s) \mathrm{d} s
\end{aligned}
$$

Then, from 4.73) and (4.76) by Sobolev inclusions we have that:

$$
\int_{0}^{t}\left\|\bar{v}_{x}^{s}(\cdot, \tau)\right\|_{L^{\infty}(\Omega)}^{2} \mathrm{~d} \tau \leq C \tau^{2} \int_{0}^{t}\|v(\cdot, s)\|_{H^{2}(\Omega)}^{2} \mathrm{~d} s
$$

Next, with the help of 4.72 we deduce

$\left\|\int_{0}^{t} \mathcal{G}(\cdot, s) \mathrm{d} s\right\|_{L^{2}(\Omega)} \leq C K \sup _{0 \leq \tau \leq t}\left\|u_{x x}(\cdot, \tau)\right\|_{L^{2}(\Omega)} \int_{0}^{t}\|v(\cdot, s)\|_{H^{2}(\Omega)}^{2} \mathrm{~d} s \int_{0}^{+\infty}\left|a^{\prime}(\tau)\right| \tau^{2} \mathrm{~d} \tau$

that is

$$
\left\|\int_{0}^{t} \mathcal{G}(\cdot, s) \mathrm{d} s\right\|_{L^{2}(\Omega)} \leq C \mathcal{E}^{3 / 2}(t)
$$


Next, let $\mathcal{G}_{x}(x, t)=I_{1}+I_{2}$, where

$$
\begin{gathered}
I_{1}=\int_{0}^{+\infty} a^{\prime}(s) g^{\prime \prime}\left(\bar{v}_{x}^{t}(s)\right)\left|\bar{v}_{x x}^{t}(s)\right|^{2} \mathrm{~d} s \\
I_{2}=\int_{0}^{+\infty} a^{\prime}(s)\left[g^{\prime}\left(\bar{v}_{x}^{t}(s)\right)-g^{\prime}(0)\right] \bar{v}_{x x x}^{t}(s) \mathrm{d} s
\end{gathered}
$$

and also $\int_{0}^{t} \mathcal{G}_{x}(x, s) \mathrm{d} s=I_{3}+I_{4}$, where

$$
\begin{gathered}
I_{3}=\int_{0}^{t} \int_{0}^{+\infty} a^{\prime}(\tau) g^{\prime \prime}\left(\bar{v}_{x}^{s}(\tau)\right)\left|\bar{v}_{x x}^{s}(\tau)\right|^{2} \mathrm{~d} \tau \mathrm{d} s \\
I_{4}=\int_{0}^{t} \int_{0}^{+\infty} a^{\prime}(\tau)\left[g^{\prime}\left(\bar{v}_{x}^{s}(\tau)\right)-g^{\prime}(0)\right] \bar{v}_{x x x}^{s}(\tau) \mathrm{d} \tau \mathrm{d} s
\end{gathered}
$$

Since $\bar{v}^{t}(s)=u(t)-u(t-s)$, using again part (i) in Lemma 4.3 we obtain

$$
\begin{aligned}
\left\|I_{1}\right\|_{L^{2}(\Omega)} & \leq 2 K \nu(t) \int_{0}^{+\infty}\left|a^{\prime}(s)\right| r_{0}(s)\left[\left\|u_{x x}^{2}(\cdot, t)\right\|_{L^{2}(\Omega)}+\left\|u_{x x}^{2}(\cdot, t-s)\right\|_{L^{2}(\Omega)}\right] \mathrm{d} s \\
& \leq 4 K \nu(t) \sup _{0 \leq s \leq t}\left\|u_{x x}(\cdot, s)\right\|_{L^{4}(\Omega)}^{2} \int_{0}^{+\infty}\left|a^{\prime}(s)\right| r_{0}(s) \mathrm{d} s
\end{aligned}
$$

This gives further down by Sobolev inclusion:

$$
\left\|I_{1}\right\|_{L^{2}(\Omega)} \leq 4 K\left(\int_{0}^{+\infty}\left|a^{\prime}(s)\right| r_{0}(s) \mathrm{d} s\right) \nu(t) \mathcal{E}(t)
$$

Next, as in (4.70), one easily obtains that

$$
\left\|I_{2}\right\|_{L^{2}(\Omega)} \leq 2 K\left(\int_{0}^{+\infty}\left|a^{\prime}(s)\right| r_{0}(s) \mathrm{d} s\right) \nu(t) \sqrt{\mathcal{E}(t)}
$$

Moreover,

$\left\|I_{3}\right\|_{L^{2}(\Omega)} \leq$

$K \int_{0}^{t} \int_{0}^{+\infty}\left|a^{\prime}(\tau)\right|\left\|\bar{v}_{x}^{s}(\cdot, \tau)\right\|_{L^{\infty}(\Omega)}\left\|u_{x x}(\cdot, s)-u_{x x}(\cdot, s-\tau)\right\|_{L^{\infty}(\Omega)}\left\|\bar{v}_{x x}^{s}(\cdot, \tau)\right\|_{L^{2}(\Omega)} \mathrm{d} \tau \mathrm{d} s$

As in the proof of 4.67) we have the following estimates:

$$
\begin{aligned}
& \left\|\bar{v}_{x}^{s}(\tau)\right\|_{L^{\infty}(\Omega)} \leq 2 \tau \mathcal{M}\left(\left\|\tilde{v}_{x}\right\|_{L^{\infty}(\Omega)}\right)(s) \\
& \left\|\bar{v}_{x x}^{s}(\tau)\right\|_{L^{2}(\Omega)} \leq 2 \tau \mathcal{M}\left(\left\|\tilde{v}_{x x}\right\|_{L^{2}(\Omega)}\right)(s)
\end{aligned}
$$

which give

$$
\left\|I_{3}\right\|_{L^{2}(\Omega)} \leq 8 K \sup _{0 \leq s \leq t}\left\|u_{x x}(\cdot, s)\right\|_{L^{\infty}(\Omega)} \int_{0}^{+\infty}\left|a^{\prime}(\tau)\right| \tau^{2} \mathrm{~d} \tau
$$




$$
\sqrt{\int_{0}^{t} \mathcal{M}\left(\left\|\tilde{v}_{x}\right\|_{L^{\infty}(\Omega)}\right)^{2}(s) \mathrm{d} s} \sqrt{\int_{0}^{t} \mathcal{M}\left(\left\|\tilde{v}_{x x}\right\|_{L^{2}(\Omega)}\right)^{2}(s) \mathrm{d} s}
$$

Using again the maximal inequality from [40] and the Sobolev embeddings leads to

$$
\left\|I_{3}\right\|_{L^{2}(\Omega)} \leq C \sup _{0 \leq s \leq t}\|u(\cdot, s)\|_{H^{3}(\Omega)} \int_{0}^{t}\|v(\cdot, s)\|_{H^{2}(\Omega)}^{2} \mathrm{~d} s
$$

that is

$$
\left\|I_{3}\right\|_{L^{2}(\Omega)} \leq C \mathcal{E}^{3 / 2}(t)
$$

Finally, for $I_{4}$ we proceed as for obtaining 4.79 and get

$$
\left\|I_{4}\right\|_{L^{2}(\Omega)} \leq C \mathcal{E}^{3 / 2}(t)
$$

The above estimates lead to the below ones:

$$
\begin{gathered}
\left\|\mathcal{G}_{x}(\cdot, t)\right\|_{L^{2}(\Omega)} \leq C \nu(t)(\mathcal{E}(t)+\sqrt{\mathcal{E}(t)}) \\
\left\|\int_{0}^{t} \mathcal{G}_{x}(\cdot, s) \mathrm{d} s\right\|_{L^{2}(\Omega)} \leq C \mathcal{E}^{3 / 2}(t)
\end{gathered}
$$

\section{Step 2.}

From 4.65 we obtain:

$$
\begin{aligned}
& \left\|u_{x x}(\cdot, t)\right\|_{L^{2}(\Omega)} \leq \frac{1}{\left|g^{\prime}(0)\right|}\left\{\frac{1}{a(0)}\left[\|f(\cdot, t)\|_{L^{2}(\Omega)}+\|\mathcal{G}(\cdot, t)\|_{L^{2}(\Omega)}+\left\|v_{t}(\cdot, t)\right\|_{L^{2}(\Omega)}\right]\right. \\
& +\left\|A_{1}\right\|_{L^{1}\left(\mathbb{R}_{+}\right)} \sup _{0 \leq s \leq t}\left[\|f(\cdot, s)\|_{L^{2}(\Omega)}+\|\mathcal{G}(\cdot, s)\|_{L^{2}(\Omega)}+\left\|v_{t}(\cdot, s)\right\|_{L^{2}(\Omega)}\right] \\
& \left.+\left\|A_{2}\right\|_{L^{1}\left(\mathbb{R}_{+}\right)} \sup _{0 \leq s \leq t}\left[\left\|\int_{0}^{s} f(\cdot, \tau) \mathrm{d} \tau\right\|_{L^{2}(\Omega)}+\left\|\int_{0}^{s} \mathcal{G}(\cdot, \tau) \mathrm{d} \tau\right\|_{L^{2}(\Omega)}+\|v(\cdot, s)\|_{L^{2}(\Omega)}+\left\|v_{0}\right\|_{L^{2}(\Omega)}\right]\right\}
\end{aligned}
$$

Using now 4.70) and 4.79 and the fact that $\nu(t)$ and $\mathcal{E}(t)$ are increasing functions we obtain (4.67). Next, (4.68) is obtained in a similar manner: one produces an equality like that of (4.94) satisfied by $\left\|u_{x x x}(\cdot, t)\right\|_{L^{2}(\Omega)}$ with $f_{x}, \mathcal{G}_{x}, v_{t x}, v_{x}, v_{0}^{\prime}$ in place of $f, \mathcal{G}, v_{t}, v, v_{0}$. Using 4.92 and 4.93 we get 4.68 .

\subsection{Smallness estimates.}

The next Proposition proves the uniform boundedness of $\mathcal{E}(t)$.

Proposition 4.2. There exist two numbers $\overline{\mathcal{E}}>0$ and $\delta>0$ independent of $n$ such that, whenever $v_{0}$ and $f$ verify $F(f)+V_{0}\left(v_{0}\right) \leq \delta$, one has

$$
\mathcal{E}(t) \leq \frac{\overline{\mathcal{E}}}{2}, \forall t \in[0, T)
$$


Proof. Remark first that, capitalizing on (4.29) and (4.9), one has $v_{t}(x, 0)=f(x, 0), v_{x t}(x, 0)=$ $f_{x}(x, 0), v_{t t}(x, 0)=-g^{\prime}(0) a(0) v_{0}^{\prime \prime}(x)+f_{t}(x, 0)$. From the definition of $\mathcal{E}(t)$ we deduce

$$
\mathcal{E}(0) \leq\left[1+2 a^{2}(0)\left|g^{\prime}(0)\right|^{2}\right]\left\|v_{0}\right\|_{H^{2}(\Omega)}^{2}+\int_{\Omega}\left[f^{2}(x, 0)+f_{x}^{2}(x, 0)+2 f_{t}^{2}(x, 0)\right] \mathrm{d} x
$$

Therefore

$$
\mathcal{E}(0) \leq 2\left[1+a^{2}(0)\left|g^{\prime}(0)\right|^{2}\right]\left(F+V_{0}\right)
$$

We now use the fact that the seminorm $w \in H^{2}(\Omega) \mapsto\left\|w_{x x}\right\|_{L^{2}(\Omega)}$ is a norm on $H^{2}(\Omega) \cap$ $H_{0}^{1}(\Omega)$, equivalent to the usual norm in $H^{2}(\Omega)$. We shall as well make use of the inequality $\left(\sqrt{V_{0}}+\sqrt{F}\right) \sqrt{\mathcal{E}(t)} \leq \eta \mathcal{E}(t)+\frac{1}{2 \eta}\left(V_{0}+F\right)$, with $\eta>0$ small enough.

From Lemmas $4.7,4.8$ and 4.10 we deduce

$$
\mathcal{E}(t) \leq C\left\{V_{0}+F+\left[\nu(t)+\nu^{3}(t)\right] \mathcal{E}(t)+\sqrt{V_{0}} \mathcal{E}(t)+\mathcal{E}^{3}(t)+\nu^{2}(t) \mathcal{E}^{2}(t)\right\}
$$

provided 4.10) holds true.

Recall also the inequality 4.7):

$$
\nu(t) \leq c_{\Omega} \sqrt{\mathcal{E}(t)}, \forall t \in[0, T)
$$

Then, we deduce from 4.98 that

$$
\mathcal{E}(t) \leq c_{1}\left[V_{0}+F+\mathcal{E}^{3}(t)\right]
$$

with $c_{1}>0$ a constant independent of $n$.

Now observe that we can choose $\overline{\mathcal{E}}>0$ and $\delta>0$ such that

$$
\left\{\begin{array}{l}
c_{1} \overline{\mathcal{E}}^{2} \leq \frac{1}{2} \\
\overline{\mathcal{E}}<\frac{\theta^{2}}{4 C_{\Omega}} \\
c_{1} \delta \leq \frac{\overline{\mathcal{E}}}{4} \\
2\left[1+a^{2}(0)\left|g^{\prime}(0)\right|^{2}\right] \delta \leq \frac{\overline{\mathcal{E}}}{2}
\end{array}\right.
$$

Let us now prove that, for any $t \in[0, T)$, (4.95) holds true. Indeed, if the contrary were true, then invoking the continuity w.r.t. time there exists $t_{2} \in(0, T)$ s.t. $\mathcal{E}(t) \leq \overline{\mathcal{E}}$, for any $t \in\left(0, t_{2}\right)$, but inequality (4.95) is false on an interval $\left(t_{1}, t_{2}\right)$ with $0<t_{1}<t_{2}$. From the second inequality in 4.101) we deduce that 4.100 is satisfied on $\left[0, t_{2}\right]$. Using once more 4.101) one gets $\mathcal{E}(t) \leq \frac{\mathcal{E}(t)}{2}+\frac{\overline{\mathcal{E}}}{4}$ which triggers $\mathcal{E}(t) \leq \frac{\overline{\mathcal{E}}}{2}$ on $\left[0, t_{2}\right]$, hence a contradiction. This later fact ends the proof.

\section{Proof of the main result.}

Remark that from Proposition 4.2 we actually deduce that for $v_{n}$ - solution of $\left(P_{n}\right)_{1},\left(P_{n}\right)_{2}$, $\left(P_{n}\right)_{3}$ - we have the following upper bounds: 


$$
\begin{aligned}
& \sup _{t \in\left[0, T_{n}\right)}\left[\left\|u_{n}(\cdot, t)\right\|_{H^{3}(\Omega)}^{2}+\left\|\left(u_{n}\right)_{t}(\cdot, t)\right\|_{H^{2}(\Omega)}^{2}+\left\|\left(u_{n}\right)_{t t}(\cdot, t)\right\|_{H^{1}(\Omega)}^{2}+\left\|\left(u_{n}\right)_{t t t}(\cdot, t)\right\|_{L^{2}(\Omega)}^{2}\right] \\
& +\int_{0}^{T_{n}}\left\{\left\|v_{n}(\cdot, t)\right\|_{H^{2}(\Omega)}^{2}+\left\|\left(v_{n}\right)_{t}(\cdot, t)\right\|_{H^{1}(\Omega)}^{2}+\left\|\left(v_{n}\right)_{t t}(\cdot, t)\right\|_{L^{2}(\Omega)}^{2}\right\} \mathrm{d} t \leq \frac{\overline{\mathcal{E}}}{2}
\end{aligned}
$$

and

$$
\sup _{\substack{x \in \Omega \\ 0<s<t<T_{n}}}\left|\int_{t-s}^{t}\left(v_{n}\right)_{x}(x, \tau) \mathrm{d} \tau\right| \leq \theta
$$

We then deduce from Proposition 4.1 that $T_{n}=+\infty$, so (5.1) and (5.2) are valid upon replacing $T_{n}$ by $+\infty$. It follows that there exist two limits

$$
u \in \bigcap_{m=0}^{3} W^{m, \infty}\left((0,+\infty) ; H^{3-m}(\Omega)\right)
$$

and

$$
v \in\left\{\bigcap_{m=0}^{2} W^{m, \infty}\left((0,+\infty) ; H^{2-m}(\Omega)\right)\right\} \cap\left\{\bigcap_{m=0}^{2} W^{m, 2}\left((0,+\infty) ; H^{2-m}(\Omega)\right)\right\}
$$

with $u(x, t)=\int_{0}^{t} v(x, s) d s$ s.t. (up to a subsequence of $n$ ) we have

$$
\frac{d^{m} u_{n}}{d t^{m}} \rightarrow \frac{d^{m} u}{d t^{m}} \quad \text { weakly } * \text { in } L^{\infty}\left((0,+\infty) ; H^{3-m}(\Omega)\right), \quad m=0,1,2,3
$$

and

$$
\frac{d^{m} v_{n}}{d t^{m}} \rightarrow \frac{d^{m} v}{d t^{m}} \quad \text { weakly in } L^{2}\left((0,+\infty) ; H^{2-m}(\Omega)\right), \quad m=0,1,2 .
$$

By the trace theorem we have $v=0$ for $x \in \partial \Omega, t \geq 0$, and $v(x, 0)=v_{0}(x)$, for $x \in \Omega$. Now remark that the equation $\left(P_{n}\right)_{1}$ can be written in the form

$$
\left(v_{n}\right)_{t}(x, t)=-\frac{\partial}{\partial x} \int_{0}^{t} a_{n}(t-s) g^{\prime}\left(\left(u_{n}\right)_{x}(x, t)-\left(u_{n}\right)_{x}(x, s)\right)\left(v_{n}\right)_{x}(x, s) \mathrm{d} s+f(x, t)
$$

We now pass to the limit in (5.3) above, for any fixed $t \geq 0$. By the trace theorem it is clear that $\left(v_{n}\right)_{t}(\cdot, t) \underset{n \rightarrow+\infty}{\stackrel{L^{2}(\Omega)}{\longrightarrow}} v_{t}(\cdot, t)$ weakly. Next, we take on to proving that

$$
\int_{0}^{t} a_{n}(t-s) g^{\prime}\left(\left(u_{n}\right)_{x}(x, t)-\left(u_{n}\right)_{x}(x, s)\right)\left(v_{n}\right)_{x}(x, s) \mathrm{d} s
$$

weakly converges in $L^{2}(\Omega)$ towards

$$
\int_{0}^{t} a(t-s) g^{\prime}\left(u_{x}(x, t)-u_{x}(x, s)\right) v_{x}(x, s) \mathrm{d} s
$$

Let $\phi \in L^{2}(\Omega)$ be fixed; we have to prove that

$$
E_{n} \underset{n \rightarrow+\infty}{\longrightarrow} E
$$


where

$$
\begin{gathered}
E_{n}=\int_{Q_{t}} \phi(x) a_{n}(t-s) g^{\prime}\left(\left(u_{n}\right)_{x}(x, t)-\left(u_{n}\right)_{x}(x, s)\right)\left(v_{n}\right)_{x}(x, s) \mathrm{d} x \mathrm{~d} s \\
E=\int_{Q_{t}} \phi(x) a(t-s) g^{\prime}\left(u_{x}(x, t)-u_{x}(x, s)\right) v_{x}(x, s) \mathrm{d} x \mathrm{~d} s
\end{gathered}
$$

By Sobolev compact inclusion we have that $\left(u_{n}\right)_{x} \underset{n \rightarrow+\infty}{\stackrel{C\left(\overline{Q_{t}}\right)}{\longrightarrow}} u_{x}$ strongly and $\left(u_{n}\right)_{x}(\cdot, t) \underset{n \rightarrow+\infty}{\stackrel{C(\bar{\Omega})}{\longrightarrow}} u_{x}(\cdot, t)$ also strongly. From $(5.2)$, with $T_{n}=+\infty$ we deduce

$$
\sup _{\substack{x \in \Omega \\ 0<s<t}}\left|\int_{t-s}^{t} v_{x}(x, \tau) \mathrm{d} \tau\right| \leq \theta
$$

Making use of 4.2 leads to the strong convergence

$$
g^{\prime}\left(\left(u_{n}\right)_{x}(x, t)-\left(u_{n}\right)_{x}(x, s)\right) \underset{n \rightarrow+\infty}{\stackrel{C\left(\overline{Q_{t}}\right)}{\longrightarrow}} g^{\prime}\left(u_{x}(x, t)-u_{x}(x, s)\right) .
$$

Since $\left(v_{n}\right)_{x} \underset{n \rightarrow+\infty}{\stackrel{L^{2}\left(Q_{t}\right)}{\longrightarrow}} v_{x}$ strongly and $a_{n} \underset{n \rightarrow+\infty}{\stackrel{L^{2}(0, t)}{\longrightarrow}} a$ strongly (consequence of assumption $\left(a_{2}\right)$ ), one easily gets (5.4) which ends the proof of Theorem 2.1.

\section{A class of totally monotone functions compliant with hypotheses $\left(a_{1}\right)$ to $\left(a_{5}\right)$.}

The goal here is to introduce a large class of functions $a$ compliant with assumptions $\left(a_{1}\right)-\left(a_{5}\right)$. The following Lemma gives sufficiently weak enough conditions so that $\left(a_{5}\right)$ holds.

Lemma 6.1. Assume that $b \in W^{1,1}(0,+\infty)$ satisfies the following conditions

(i) $t b^{\prime} \in L^{1}(0,+\infty)$

(ii) there exists $M_{4}>0$ and $\alpha_{1}>0$ s.t. $|\mathcal{F} b(\omega)| \geq \frac{M_{4}}{1+|\omega|^{\alpha_{1}}}, \forall \omega \in \mathbb{R}$

(iii) there exists $M_{5}>0$ and $\alpha_{2}>0$ s.t. $\left|\mathcal{F} b^{\prime}(\omega)\right| \leq \frac{M_{5}}{1+|\omega|^{\alpha_{2}}}, \forall \omega \in \mathbb{R}$

(iv) there exists $\alpha_{3} \in \mathbb{R}$ s.t. the function $\mathbb{R} \ni t \mapsto t b(t) \in \mathbb{R}$ is an element of $H^{\alpha_{3}}(\mathbb{R})$

Then there exists $M_{6}>0$ depending only on $M_{4}, M_{5}, \alpha_{1}, \alpha_{2}$ and $\alpha_{3}$, and $p \in \mathbb{N}^{*}$ depending only on $\alpha_{1}$ and $\alpha_{2}$ and $\alpha_{3}$, s.t.

$$
\frac{\left(\mathcal{F} b^{\prime}\right)^{p}}{\mathcal{F} b} \in \mathcal{F}\left(B_{L^{1}(\mathbb{R})}\left(0, M_{7}\right)\right)
$$

where

$$
M_{7}=M_{6}\left[1+\left\|t b^{\prime}\right\|_{L^{1}(\mathbb{R})}+\|t b\|_{H^{\alpha_{3}(\mathbb{R})}}\right]
$$


Proof. Since $H^{1}(\mathbb{R}) \subset \mathcal{F} L^{1}(\mathbb{R})$ and $\left\|\mathcal{F}^{-1} w\right\|_{L^{1}(\mathbb{R})} \leq C\|w\|_{H^{1}(\mathbb{R})}, \forall w \in H^{1}(\mathbb{R})$ (see [29]), it suffices to consider the $H^{1}$ norm of $E \equiv \frac{\left[\mathcal{F} b^{\prime}\right]^{p}}{\mathcal{F} b}$. From hypotheses (ii) and (iii) it is clear that, for $p$ large enough depending on $\alpha_{1}$ and $\alpha_{2}$, we have

$$
\|E\|_{L^{2}(\mathbb{R})} \leq M_{6}
$$

where $M_{6}$ depends on $M_{4}, M_{5}$ and $\alpha_{2}$. We also have $E^{\prime}=E_{1}-E_{2}$, with

$$
\begin{gathered}
E_{1}:=p \frac{\left[\mathcal{F} b^{\prime}\right]^{p-1}\left[\mathcal{F} b^{\prime}\right]^{\prime}}{\mathcal{F} b} \\
E_{2}:=\frac{\left[\mathcal{F} b^{\prime}\right]^{p}[\mathcal{F} b]^{\prime}}{(\mathcal{F} b)^{2}}
\end{gathered}
$$

Since $\left|\left(\mathcal{F} b^{\prime}\right)^{\prime}\right|=\left|\mathcal{F}\left(t b^{\prime}\right)\right| \in L^{\infty}\left(\mathbb{R}_{+}\right)$, from the above mentioned assumptions we get there exists $p$ large enough depending on $\alpha_{1}$ and $\alpha_{2}$ s.t.

$$
\left\|E_{1}\right\|_{L^{2}(\mathbb{R})} \leq M_{6}\left\|t b^{\prime}\right\|_{L^{1}(\mathbb{R})}
$$

From assumption (iv) and the fact that $\left|(\mathcal{F} b)^{\prime}\right|=|\mathcal{F}(t b)|$ we have that the function $\omega \longrightarrow$ $\left(1+\omega^{2}\right)^{\alpha_{3} / 2}(\mathcal{F} b)^{\prime}(\omega) \in L^{2}(\mathbb{R})$, and, $\left\|\left(1+\omega^{2}\right)^{\alpha_{3} / 2}(\mathcal{F} b)^{\prime}(\omega)\right\|_{L^{2}(\mathbb{R})}=\|t b\|_{H^{\alpha_{3}(\mathbb{R})}}$.

Then there exists $p$ large enough depending on $\alpha_{1}, \alpha_{2}$ and $\alpha_{3}$ s.t.

$$
\left\|E_{2}\right\|_{L^{2}(\mathbb{R})} \leq M_{6}\|t b\|_{H^{\alpha}(\mathbb{R})}
$$

with $M_{6}$ as before. From (6.3), (6.6) and (6.7) the claimed result follows.

Let $\mu$ be a positive, finite and non-zero Borel measure on $\mathbb{R}_{+}$, satisfying

$\left(\mu_{1}\right)$ : the function $\mathbb{R}_{+} \ni \rho \mapsto \frac{1}{\rho^{2}}$ is an element of $L_{\mu}^{1}(0,+\infty)$

$\left(\mu_{2}\right)$ : there exists $\gamma \in(0,1)$ s.t. the function $\mathbb{R}_{+} \ni \rho \mapsto \rho^{\gamma}$ is an element of $L_{\mu}^{1}(0,+\infty)$

Remark that, as a consequence of these hypotheses, the function $\mathbb{R}_{+} \ni \rho \mapsto \rho^{\beta}$ is an element of $L_{\mu}^{1}(0,+\infty)$ for any $\beta \in[-2, \gamma]$.

We now consider the following totally monotone function (see [36])

$$
\tilde{a}:[0,+\infty) \rightarrow \mathbb{R}, \tilde{a}(t)=\int_{\mathbb{R}_{+}} e^{-\rho t} \mathrm{~d} \mu(\rho), \forall t \geq 0
$$

This Section main result is contained in the below theorem:

Theorem 6.1. Assume the hypotheses $\left(\mu_{1}\right)$ and $\left(\mu_{2}\right)$ hold true. Then the function $\tilde{a}$ given by (6.8) satisfies the hypotheses $\left(a_{1}\right)-\left(a_{5}\right)$ of Section 2 with

$$
\tilde{a}_{n}(t)=\int_{[0, n)} e^{-\rho t} \mathrm{~d} \mu(\rho), \forall t \geq 0, \forall n \in \mathbb{N}^{*}
$$


Proof. Since the measure $\mu$ is finite, it is clear that $\tilde{a}_{n} \in \mathscr{C}^{\infty}\left(\mathbb{R}_{+}\right)$, and for any $t \in \mathbb{R}_{+}$and $k \in \mathbb{N},\left(\tilde{a}_{n}\right)^{(k)}(t)=\int_{[0, n)}(-1)^{k} \rho^{k} e^{-\rho t} \mathrm{~d} \mu(\rho)$. This gives $\tilde{a}_{n} \in W^{p, \infty}(0,+\infty)$, for any $p \in \mathbb{N}$ and also $\tilde{a}_{n}^{\prime}<0$.

Let $k \in \mathbb{N}$ and $q \in \mathbb{R}_{+}$. Then

$$
\int_{0}^{+\infty} t^{q}\left(\tilde{a}_{n}\right)^{(k)}(t) \mathrm{d} t=(-1)^{k} \int_{0}^{+\infty} t^{q} \int_{[0, n)} \rho^{k} e^{-\rho t} \mathrm{~d} \mu(\rho) \mathrm{d} t=(-1)^{k} \int_{[0, n)} \rho^{k}\left(\int_{0}^{+\infty} t^{q} e^{-\rho t} \mathrm{~d} t\right) \mathrm{d} \mu(\rho)
$$

Taking $\tau=\rho t$ in the integral w.r.t. $t$ leads to

$$
\int_{0}^{+\infty} t^{q}\left|\left(\tilde{a}_{n}\right)^{(k)}(t)\right| \mathrm{d} t=\int_{0}^{+\infty} \tau^{q} e^{-\tau} \mathrm{d} \tau \int_{[0, n)} \rho^{k-q-1} \mathrm{~d} \mu(\rho)
$$

Invoking hypotheses $\left(\mu_{1}\right)$ and $\left(\mu_{2}\right)$ gives

$$
\int_{[0,+\infty)} \rho^{k-q-1} \mathrm{~d} \mu(\rho)<\infty
$$

provided that

$$
0 \leq q+1-k \leq 2
$$

For $q=0$ and $k=0$ or $k=1$ one sees that 6.11) is verified, therefore $\left(a_{1}\right)$ and $\left(a_{2}\right)$ are valid.

For $q=2$ and $k=16$ (6.11) is also verified, then $\int_{0}^{+\infty} t^{2}\left|\tilde{a}_{n}^{\prime}(t)\right| \mathrm{d} t$ is bounded. The same for $q=1$ and $k=2$, with this time $\int_{0}^{+\infty} t\left|\tilde{a}_{n}^{\prime \prime}(t)\right| \mathrm{d} t$ bounded. The later grants $\left(a_{3}\right)$ is valid.

Next, by Fubini's theorem we obtain, for $\omega \in \mathbb{R}$,

$$
\mathcal{F} \tilde{a}_{n}(\omega)=\int_{0}^{+\infty} \int_{[0, n)} e^{-\rho t} \mathrm{~d} \mu(\rho) e^{-i \omega t} \mathrm{~d} t=\int_{[0, n)} \frac{\mathrm{d} \mu(\rho)}{\rho+i \omega}
$$

from which one gets

$$
\operatorname{Re}\left[\mathcal{F} \tilde{a}_{n}(\omega)\right]=\int_{[0, n)} \frac{\rho}{\rho^{2}+\omega^{2}} \mathrm{~d} \mu(\rho)
$$

Now, assumption $\left(\mu_{1}\right)$ gives $\mu(\{0\})=0$, so, there exists $\underline{\mu}$ and $\bar{\mu}$ s.t. $0<\underline{\mu}<\bar{\mu}$ and $\mu([\underline{\mu}, \bar{\mu}])>0$. Take $n>\bar{\mu}$ to get

$$
\operatorname{Re}\left[\mathcal{F} \tilde{a}_{n}(\omega)\right] \geq \frac{\underline{\mu}}{\bar{\mu}^{2}+\omega^{2}} \mu([\underline{\mu}, \bar{\mu}]), \forall \omega \in \mathbb{R}
$$

which proves $\left(a_{4}\right)$.

Now we prove that the hypotheses of Lemma 6.1 are verified for $b=\tilde{a}_{n}$, with constants independent of $n$.

The last inequality also proves that (ii) of Lemma 6.1 is verified with $M_{3}$ independent of $n$ and $\alpha_{1}=2$. Taking $q=k=1$ (which satisfy (6.11) ) we deduce that part (i) of Lemma 6.1 is also verified, and that $\left\|t \tilde{a}_{n}^{\prime}\right\|_{L^{1}(0,+\infty)}$ is bounded.

Next, on one hand, we easily calculate

$$
\mathcal{F} \tilde{a}_{n}^{\prime}(\omega)=-\int_{[0, n)} \frac{\rho}{\rho+i \omega} \mathrm{d} \mu(\rho)
$$


which gives

$$
\left|\mathcal{F} \tilde{a}_{n}^{\prime}(\omega)\right| \leq \int_{[0, n)} \frac{\rho}{\sqrt{\rho^{2}+\omega^{2}}} \mathrm{~d} \mu(\rho)
$$

We deduce that

$$
\left|\mathcal{F} \tilde{a}_{n}^{\prime}(\omega)\right| \leq \int_{\mathbb{R}_{+}} \mathrm{d} \mu(\rho)
$$

On the other hand now, we use the fact that

$$
\rho^{2(1-\gamma)}|\omega|^{2 \gamma} \leq \gamma|\omega|^{2}+(1-\gamma) \rho^{2} \leq|\omega|^{2}+\rho^{2}
$$

to get from 6.12 , for $\omega \neq 0$,

$$
\left|\mathcal{F} \tilde{a}_{n}^{\prime}(\omega)\right| \leq \int_{[0, n)} \frac{\rho}{\rho^{1-\gamma}|\omega|^{\gamma}} \mathrm{d} \mu(\rho)=\frac{1}{|\omega|^{\gamma}} \int_{[0, n)} \rho^{\gamma} \mathrm{d} \mu(\rho)
$$

Invoke $\left(\mu_{2}\right)$ to get, for $\omega \neq 0$,

$$
\left|\mathcal{F} \tilde{a}_{n}^{\prime}(\omega)\right| \leq \frac{1}{|\omega|^{\gamma}} \int_{\mathbb{R}_{+}} \rho^{\gamma} \mathrm{d} \mu(\rho)
$$

Then, 6.13 and 6.14 give

$$
\left|\mathcal{F} \tilde{a}_{n}^{\prime}(\omega)\right| \leq \frac{2}{1+|\omega|^{\gamma}} \int_{\mathbb{R}_{+}}\left(1+\rho^{\gamma}\right) \mathrm{d} \mu(\rho)
$$

Then the assumption formulated in (iii) of Lemma 6.1 is verified with $\alpha_{2}=\gamma$ and a constant $M_{4}$ independent of $n$.

Finally, the inequality (6.11) is verified with $q=1$ and $k=0$. From $(6.9)$ and assumption $\left(\mu_{2}\right)$ we get

$$
\left\|t \tilde{a}_{n}\right\|_{L^{1}\left(\mathbb{R}_{+}\right)} \leq \int_{0}^{+\infty} \tau e^{-\tau} \mathrm{d} \tau \int_{\mathbb{R}_{+}} \rho^{-2} \mathrm{~d} \mu(\rho)<\infty
$$

The above entails $t \tilde{a}_{n}$ is bounded in $H^{-1}(\mathbb{R})$; consequently hypothesis (iv) of Lemma 6.1 is verified with $\beta=-1$. We then deduce that the conclusion of Lemma 6.1 is verified with a constant $M_{6}>0$ independent of $n$. Then hypothesis $\left(a_{5}\right)$ is verified.

Remark 6.1. The relaxation function of the Doi-Edwards theory,

$$
a_{D E}(t)=\sum_{k=1}^{+\infty} \frac{1}{(2 k+1)^{2}} e^{-(2 k+1)^{2} \pi^{2} D t / L^{2}}, t \geq 0,
$$

is actually a particular case of (6.8) with the measure $\mu_{D E}=\sum_{k=1}^{+\infty} \frac{1}{(2 k+1)^{2}} \delta_{(2 k+1)^{2} \pi^{2} D / L^{2}}$, where $\delta_{(2 k+1)^{2} \pi^{2} D / L^{2}}$ is Dirac's measure at $(2 k+1)^{2} \pi^{2} D / L^{2}$.

It is easy to see that the assumptions $\left(\mu_{1}\right),\left(\mu_{2}\right)$ are verified for this measure, and this paper results can be applied for the $a_{D E}$ function. 


\section{Appendix.}

\subsection{Appendix 1.}

For sake of clarity we show here how equation $(1.12$ can be solved employing the method of characteristics in a more general case, that is with $\frac{\partial v}{\partial x} \mathcal{H}_{0}(u)$ being replaced by the tensor $\mathscr{M}(t, u)$ given in $(1.3)$ with $\kappa$ an arbitrary tensor. In the end we shall make the necessary particular assumptions to explicitly obtain the solution of (1.12).

Consider the problem:

$$
\begin{gathered}
\frac{\partial F}{\partial t}=D \frac{\partial^{2} F}{\partial s^{2}}-\frac{\partial}{\partial u} \cdot[\mathscr{M}(t, u) F],(t, u, s) \in[0,+\infty) \times S_{2} \times(0, L) \\
F(s=0)=F(s=L)=\frac{1}{4 \pi} \\
F(t=0)=F_{0}
\end{gathered}
$$

Given that $\frac{\partial}{\partial u} \cdot \mathscr{M}=-3 \kappa u \cdot u \equiv-3 \kappa: u u$ one may re-write 7.1 as

$$
\frac{\partial F}{\partial t}=D \frac{\partial^{2} F}{\partial s^{2}}+(3 \kappa u \cdot u) F-\mathscr{M} \cdot \frac{\partial F}{\partial u}
$$

and further on, with $\bar{F}=F-\frac{1}{4 \pi}$ so that $\bar{F}(s=0)=\bar{F}(s=L)=0$, as

$$
\frac{\partial \bar{F}}{\partial t}-D \frac{\partial^{2} \bar{F}}{\partial s^{2}}-(3 \kappa u \cdot u) \bar{F}+\mathscr{M} \cdot \frac{\partial \bar{F}}{\partial u}=\frac{3}{4 \pi} \kappa u \cdot u
$$

We now search for solutions $\bar{F}$ in the form

$$
\bar{F}(t, u, s)=\sum_{k=1}^{+\infty} \bar{F}_{k}(t, u) \sin \frac{k \pi}{L} s
$$

Introducing the above into 7.4 gives, for any $k \in \mathbb{N}^{*}$,

$$
\frac{\partial \bar{F}_{k}}{\partial t}+\left(\frac{k^{2} \pi^{2} D}{L^{2}}-3 \kappa u \cdot u\right) \bar{F}_{k}+\mathscr{M} \cdot \frac{\partial \bar{F}_{k}}{\partial u}=\left(\frac{3}{4 \pi} \kappa u \cdot u\right) a_{k}
$$

with $a_{k}$ satisfying $(1.16)$. One gets

$$
F(t, u, s)=\sum_{k=1}^{+\infty} F_{k}(t, u) \sin \frac{k \pi}{L} s
$$

with $F_{k}$ solutions to

$$
\begin{gathered}
\frac{\partial F_{k}}{\partial t}+\left(\frac{k^{2} \pi^{2} D}{L^{2}}-3 \kappa u \cdot u\right) F_{k}+\mathscr{M} \cdot \frac{\partial F_{k}}{\partial u}=\frac{\pi}{4} \frac{D}{L^{2}} k^{2} a_{k} \\
F_{k}(t=0)=F_{0 k}
\end{gathered}
$$

with $F_{0 k}$ being given by 1.15 . As an aside, in the above $x$ is to be considered only as a parameter, not entering the calculations.

To solve (7.6)-(7.7) we use the method of characteristics. Specifically, for all $t, \tau \geq 0$, denote $M(t, \tau)$ the unique solution of the differential system 


$$
\begin{gathered}
\frac{\partial M}{\partial t}=\kappa(t) M, \\
M(t=\tau, \tau)=I d_{3}
\end{gathered}
$$

where $I d_{3}$ is the Kronecker's delta (unity) tensor. For any $w \in S_{2}$, set $\xi(t, \tau, w)=$ $\frac{M(t, \tau) w}{\|M(t, \tau) w\|} \in S_{2}$. One easily checks that

$$
\frac{\partial \xi}{\partial t}=\kappa(t) \xi-[\kappa(t) \xi \cdot \xi] \xi, t, \tau \geq 0
$$

and that

$$
\xi(t=\tau, \tau, w)=w, \tau \geq 0
$$

Equation (7.6) is now solved along the characteristic curves $\xi(t, 0, w)$. To do so, for any fixed $w \in S_{2}$, let $A_{k}(t, w)=F_{k}(t, \xi(t, 0, w))$; from (7.6) one obtains

$$
\frac{\partial A_{k}}{\partial t}=\left[3 \kappa(t) \xi(t, 0, w) \cdot \xi(t, 0, w)-\frac{k^{2} \pi^{2} D}{L^{2}}\right] A_{k}+\frac{\pi}{4} \frac{D}{L^{2}} k^{2} a_{k}
$$

with

$$
A_{k}(t=0)=F_{0 k}(w)
$$

One gets

$$
\begin{aligned}
A_{k}(t) & =\exp \left[-\frac{k^{2} \pi^{2} D}{L^{2}} t+3 \int_{0}^{t} \kappa(\tau) \xi(\tau, 0, w) \cdot \xi(\tau, 0, w) \mathrm{d} \tau\right] F_{0 k}(w) \\
& +\frac{\pi}{4} \frac{D}{L^{2}} k^{2} a_{k} \int_{0}^{t} \exp \left[-\frac{k^{2} \pi^{2} D}{L^{2}}(t-\tau)+3 \int_{0}^{t} \kappa(r) \xi(r, 0, w) \cdot \xi(r, 0, w) \mathrm{d} r\right] \mathrm{d} \tau
\end{aligned}
$$

Letting $u=\xi(t, 0, w)$ implies $\xi(\tau, 0, w)=\xi(\tau, t, u)$ (because $w=\xi(0, t, u))$. Next, for any $t_{1}, t_{2} \geq 0$,

$$
\begin{aligned}
& \int_{t_{1}}^{t_{2}} \kappa(\tau) \xi(\tau, t, u) \cdot \xi(\tau, t, u) \mathrm{d} \tau=\int_{t_{1}}^{t_{2}} \kappa(\tau) \frac{M(\tau, t) w}{\|M(\tau, t) w\|} \cdot \frac{M(\tau, t) w}{\|M(\tau, t) w\|} \mathrm{d} \tau \\
& =\int_{t_{1}}^{t_{2}} \frac{\frac{\partial}{\partial \tau}(M(\tau, t) w)}{\|M(\tau, t) w\|} \cdot \frac{M(\tau, t) w}{\|M(\tau, t) w\|} \mathrm{d} \tau=\log \left(\frac{\left\|M\left(t_{2}, t\right) u\right\|}{\left\|M\left(t_{1}, t\right) u\right\|}\right)
\end{aligned}
$$

With the help of the above and from $(7.8)$ we gets

$$
F_{k}(t, u)=\frac{e^{-k^{2} \pi^{2} D t / L^{2}}}{\|M(0, t) u\|^{3}} F_{0 k}\left(\frac{M(0, t) u}{\|M(0, t) u\|}\right)+\frac{\pi D k^{2}}{4 L^{2}} a_{k} \int_{0}^{t} \frac{e^{-k^{2} \pi^{2} D(t-\tau) / L^{2}}}{\|M(\tau, t) u\|^{3}} \mathrm{~d} \tau
$$

We conclude that the solution of (7.1), (7.2), (7.3) is given by (7.5), with $F_{k}$ from (7.9) above. In the particular case where $\mathscr{M}(t, u)=\frac{\partial v}{\partial x} \mathcal{H}_{0}(u)$ as in 1.12 , one has

$$
M(t, \tau)=I d_{3}+\left[\int_{\tau}^{t} \frac{\partial v}{\partial x}(x, r) \mathrm{d} r\right] M_{0}
$$


for all $t, \tau \geq 0$.

This allows to obtain (1.14) knowing that

$$
M(\tau, t) u=\left(u_{1}-\left[\int_{\tau}^{t} \frac{\partial v}{\partial x}(x, r) \mathrm{d} r\right] u_{2}, u_{2}, u_{3}\right)
$$

\subsection{Appendix 2.}

The task here is to prove Lemma 4.6, relabeled below as Lemma 7.2

Let the function $\xi=\xi(s, t, x)$ be defined a.e. as $\xi(s, t, x):=a^{\prime}(s)\left[g^{\prime}\left(\bar{v}_{x}^{t}(x, s)\right)-g^{\prime}(0)\right]$, $s \in[0,+\infty), t \in[0, T), x \in \Omega$. Let $D_{T}:=\{(s, t): s \in[0,+\infty), t \in[0, T), s \neq t\}$.

In the following, $\partial_{1} \xi, \partial_{2} \xi, \partial_{22} \xi$ stand for $\frac{\partial \xi}{\partial s}, \frac{\partial \xi}{\partial t}$, and $\frac{\partial^{2} \xi}{\partial t^{2}}$, respectively.

The first step is proving the following:

Lemma 7.1. Invoking the above defined notations,

(i) one has: $\xi \in \mathscr{C}^{1}\left(D_{T} ; H^{1}(\Omega)\right), \frac{\partial^{2} \xi}{\partial t^{2}} \in \mathscr{C}^{0}\left(D_{T} ; L^{2}(\Omega)\right)$;

(ii) assuming (4.10) holds true, one has the following estimates a.e. $x \in \Omega, s \in[0,+\infty)$

$$
\begin{gathered}
|\xi(s, t, x)| \leq K \nu(t)\left|a^{\prime}(s)\right| r_{0}(s) \\
\left|\frac{\partial \xi}{\partial t}(s, t, x)\right| \leq 2 K \theta \nu(t)\left|a^{\prime}(s)\right| \\
\left|\frac{\partial \xi}{\partial s}(s, t, x)\right| \leq K \nu(t)\left[\left|a^{\prime \prime}(s)\right| r_{0}(s)+\theta\left|a^{\prime}(s)\right|\right] \\
\left|\frac{\partial^{2} \xi}{\partial t^{2}}(s, t, x)\right| \leq 4 \nu^{2}(t)\left[K \theta+\left|g^{(3)}(0)\right|\right]\left|a^{\prime}(s)\right| \\
+K \nu(t)\left|a^{\prime}(s)\right| r_{0}(s)\left[\left|v_{x t}(x, t)\right|+\left|v_{x t}(x, t-s)\right|\right]
\end{gathered}
$$

The above derivatives may be considered in the classical sense, as they are defined for $s \neq t$.

Proof. Observe that

$$
\begin{gathered}
\frac{\partial \xi}{\partial t}=a^{\prime}(s) g^{\prime \prime}\left(\bar{v}_{x}^{t}(s)\right)\left[v_{x}(t)-v_{x}(t-s)\right] \\
\frac{\partial \xi}{\partial s}=a^{\prime \prime}(s)\left[g^{\prime}\left(\bar{v}_{x}^{t}(s)\right)-g^{\prime}(0)\right]+a^{\prime}(s) g^{\prime \prime}\left(\bar{v}_{x}^{t}(s)\right) v_{x}(t-s) \\
\frac{\partial^{2} \xi}{\partial t^{2}}=a^{\prime}(s) g^{(3)}\left(\bar{v}_{x}^{t}(s)\right)\left[v_{x}(t)-v_{x}(t-s)\right]^{2}+a^{\prime}(s) g^{\prime \prime}\left(\bar{v}_{x}^{t}(s)\right)\left[v_{x t}(t)-v_{x t}(t-s)\right]
\end{gathered}
$$

Repeated use of part (i) of Lemma 4.3 triggers the result.

For sake of clarity and - last but not least - reader's convenience, we restate Lemma's 4.6 content and then achieve its proof. 
Lemma 7.2. Under the assumption that 4.10 is fulfilled, one has:

$$
\begin{aligned}
\int_{\Omega} v_{t t}^{2}(x, t) \mathrm{d} x-2 g^{\prime}(0) \lim _{h \rightarrow 0_{+}} \frac{1}{h^{2}} Q\left(\triangle_{h} v_{x t}, t, a\right) \leq & C\{F+\sqrt{F} \sqrt{\mathcal{E}(t)} \\
& \left.+\left[\nu(t)+\nu^{3}(t)\right] \mathcal{E}(t)+\sqrt{V_{0}} \mathcal{E}(t)\right\}
\end{aligned}
$$

Proof. Derivate (2.4) w.r.t. $t$ and apply $\triangle_{h}$ on the resulting equation. One gets:

$$
\triangle_{h} v_{t t}=\int_{0}^{+\infty} a^{\prime}(s) \triangle_{h}\left(g\left(\bar{v}_{x}^{t}(s)\right)\right)_{x t} \mathrm{~d} s+\triangle_{h} f_{t}
$$

Multiply the above by $\triangle_{h} v_{t}$, integrate on $\Omega \times[0, t]$ to obtain

$$
\begin{aligned}
& \frac{1}{2} \int_{\Omega}\left[\triangle_{h} v_{t}(x, t)\right]^{2} \mathrm{~d} x-\frac{1}{2} \int_{\Omega}\left[\triangle_{h} v_{t}(x, 0)\right]^{2} \mathrm{~d} x \\
& =-\int_{0}^{t} \int_{\Omega} \int_{0}^{+\infty} a^{\prime}(\tau) \triangle_{h} g\left(\bar{v}_{x}^{s}(x, \tau)\right)_{s} \triangle_{h} v_{x t}(x, s) \mathrm{d} \tau \mathrm{d} x \mathrm{~d} s \\
& +\int_{0}^{t} \int_{\Omega} \triangle_{h} f_{t}(x, s) \triangle_{h} v_{t}(x, s) \mathrm{d} x \mathrm{~d} s
\end{aligned}
$$

Observing that

$$
g\left(\bar{v}_{x}^{s}(x, \tau)\right)_{s}=g^{\prime}\left(\bar{v}_{x}^{s}(x, \tau)\right)\left[v_{x}(x, s)-v_{x}(x, s-\tau)\right]
$$

leads to

$$
-\int_{0}^{t} \int_{\Omega} \int_{0}^{+\infty} a^{\prime}(\tau) \triangle_{h} g\left(\bar{v}_{x}^{s}(x, \tau)\right)_{s} \triangle_{h} v_{x t}(x, s) \mathrm{d} \tau \mathrm{d} x \mathrm{~d} s=I_{1}+I_{2}+I_{3}+I_{4}
$$

where:

$$
\begin{gathered}
I_{1}=-\int_{0}^{t} \int_{\Omega} \int_{0}^{+\infty} a^{\prime}(\tau) \triangle_{h} v_{x t}(x, s) \triangle_{h} g^{\prime}\left(\bar{v}_{x}^{s}(x, \tau)\right)\left[v_{x}(s+h)-v_{x}(s+h-\tau)\right] \mathrm{d} \tau \mathrm{d} x \mathrm{~d} s \\
I_{2}=-\int_{0}^{t} \int_{\Omega} \int_{0}^{+\infty} a^{\prime}(\tau) \triangle_{h} v_{x t}(x, s)\left[g^{\prime}\left(\bar{v}_{x}^{s}(x, \tau)\right)-g^{\prime}(0)\right] \triangle_{h} v_{x}(x, s) \mathrm{d} \tau \mathrm{d} x \mathrm{~d} s \\
I_{3}=g^{\prime}(0) \int_{0}^{t} \int_{\Omega} \int_{0}^{+\infty} a^{\prime}(\tau) \triangle_{h} v_{x t}(x, s)\left[\triangle_{h} v_{x}(s-\tau)-\triangle_{h} v_{x}(s)\right] \mathrm{d} \tau \mathrm{d} x \mathrm{~d} s \\
I_{4}=\int_{0}^{t} \int_{\Omega} \int_{0}^{+\infty} a^{\prime}(\tau) \triangle_{h} v_{x t}(x, s)\left[g^{\prime}\left(\bar{v}_{x}^{s}(x, \tau)\right)-g^{\prime}(0)\right] \triangle_{h} v_{x}(s-\tau) \mathrm{d} \tau \mathrm{d} x \mathrm{~d} s
\end{gathered}
$$

Integrating by parts w.r.t. $s$ leads to $I_{1}=I_{11}+I_{12}$, where:

$$
I_{11}=-\int_{\Omega} \int_{0}^{+\infty} a^{\prime}(\tau) \triangle_{h} v_{x}(x, t) \triangle_{h} g^{\prime}\left(\bar{v}_{x}^{t}(x, \tau)\right)\left[v_{x}(x, t+h)-v_{x}(x, t+h-\tau)\right] \mathrm{d} \tau \mathrm{d} x
$$




$$
\begin{aligned}
& +\int_{0}^{t} \int_{\Omega} \int_{0}^{+\infty} a^{\prime}(\tau) \triangle_{h} v_{x}(x, s) \triangle_{h}\left[g^{\prime \prime}\left(\bar{v}_{x}^{s}(x, \tau)\right)\left(v_{x}(x, s)-v_{x}(x, s-\tau)\right)\right] \\
& {\left[v_{x}(x, s+h)-v_{x}(x, s+h-\tau)\right] \mathrm{d} \tau \mathrm{d} x \mathrm{~d} s} \\
& +\int_{0}^{t} \int_{\Omega} \int_{0}^{+\infty} a^{\prime}(\tau) \triangle_{h} v_{x}(x, s) \triangle_{h} g^{\prime}\left(\bar{v}_{x}^{s}(x, \tau)\right)\left[v_{x t}(x, s+h)-v_{x t}(x, s+h-\tau)\right] \mathrm{d} \tau \mathrm{d} x \mathrm{~d} s
\end{aligned}
$$

and

$$
\begin{aligned}
I_{12} & =\int_{\Omega} \int_{0}^{+\infty} a^{\prime}(\tau) \triangle_{h} v_{x}(0) \triangle_{h} g^{\prime}\left(\bar{v}_{x}^{0}(x, \tau)\right)\left[v_{x}(x, h)-v_{x}(x, h-\tau)\right] \mathrm{d} \tau \mathrm{d} x \\
& -\int_{0}^{t} \int_{\Omega} a^{\prime}(s+h) \triangle_{h} g^{\prime}\left(\int_{0}^{s} v_{x}(x, \lambda) \mathrm{d} \lambda\right) v_{0}^{\prime}(x) \triangle_{h} v_{x}(x, s) \mathrm{d} x \mathrm{~d} s
\end{aligned}
$$

Observe that

$$
\begin{aligned}
& \int_{\Omega} \int_{0}^{+\infty} a^{\prime}(\tau) \triangle_{h} v_{x}(0) \triangle_{h} g^{\prime}\left(\bar{v}_{x}^{0}(x, \tau)\right)\left[v_{x}(x, h)-v_{x}(x, h-\tau)\right] \mathrm{d} \tau \mathrm{d} x \\
& =\int_{\Omega}\left[v_{x}(h)-v_{x}(0)\right] \int_{0}^{h} a^{\prime}(\tau)\left[g^{\prime}\left(\int_{h-\tau}^{h} v_{x}(\lambda) \mathrm{d} \lambda\right)-g^{\prime}(0)\right]\left[v_{x}(h)-v_{x}(h-\tau)\right] \mathrm{d} \tau \\
& -\int_{\Omega} a(h)\left[v_{x}(h)-v_{x}(0)\right]\left[g^{\prime}\left(\int_{0}^{h} v_{x}(\lambda) \mathrm{d} \lambda\right)-g^{\prime}(0)\right] v_{x}(h) \mathrm{d} x
\end{aligned}
$$

By integrating the first term by parts w.r.t. $\tau$ one gets

$$
\begin{aligned}
I_{12} & =\int_{\Omega}\left[v_{x}(h)-v_{x}(0)\right]^{2} a(h)\left[g^{\prime}\left(\int_{0}^{h} v_{x}(\lambda) \mathrm{d} \lambda\right)-g^{\prime}(0)\right] \mathrm{d} x \\
& -\int_{\Omega}\left[v_{x}(h)-v_{x}(0)\right] \int_{0}^{h} a(\tau) g^{\prime \prime}\left(\int_{h-\tau}^{h} v_{x}(\lambda) \mathrm{d} \lambda\right) v_{x}(h-\tau)\left[v_{x}(h)-v_{x}(h-\tau)\right] \mathrm{d} \tau \mathrm{d} x \\
& -\int_{\Omega}\left[v_{x}(h)-v_{x}(0)\right] \int_{0}^{h} a(\tau)\left[g^{\prime}\left(\int_{h-\tau}^{h} v_{x}(\lambda) \mathrm{d} \lambda\right)-g^{\prime}(0)\right] v_{x t}(h-\tau) \mathrm{d} \tau \mathrm{d} x \\
& -\int_{\Omega} a(h)\left[v_{x}(h)-v_{x}(0)\right]\left[g^{\prime}\left(\int_{0}^{h} v_{x}(\lambda) \mathrm{d} \lambda\right)-g^{\prime}(0)\right] v_{x}(h) \mathrm{d} x \\
& -\int_{0}^{t} \int_{\Omega} a^{\prime}(s+h) \triangle_{h} g^{\prime}\left(\int_{0}^{s} v_{x}(\lambda) \mathrm{d} \lambda\right) v_{0}^{\prime}(x) \triangle_{h} v_{x}(s) \mathrm{d} x \mathrm{~d} s
\end{aligned}
$$

Next, dividing the above by $h^{2}$, passing to the limit for $h \rightarrow 0_{+}$and using the fact that $v$ and its derivatives up to order 2 belong to $\mathscr{C}^{2}\left([0, T) ; L^{2}(\Omega)\right)$ leads to

$$
\frac{1}{h^{2}} I_{1} \underset{h \rightarrow 0_{+}}{\longrightarrow} J_{1}+J_{01}
$$

where

$$
\begin{aligned}
J_{1}= & -\int_{\Omega} \int_{0}^{+\infty} \partial_{2} \xi(\tau, t, x) v_{x t}(x, t)\left[v_{x}(x, t)-v_{x}(x, t-\tau)\right] \mathrm{d} \tau \mathrm{d} x \\
& +\int_{0}^{t} \int_{\Omega} \int_{0}^{+\infty} \partial_{22} \xi(\tau, s, x) v_{x t}(x, s)\left[v_{x}(x, s)-v_{x}(x, s-\tau)\right] \mathrm{d} \tau \mathrm{d} x \mathrm{~d} s
\end{aligned}
$$




$$
+\int_{0}^{t} \int_{\Omega} \int_{0}^{+\infty} \partial_{2} \xi(\tau, s, x) v_{x t}(x, s)\left[v_{x t}(x, s)-v_{x t}(x, s-\tau)\right] \mathrm{d} \tau \mathrm{d} x \mathrm{~d} s
$$

and

$$
J_{01}=-\int_{0}^{t} \int_{\Omega} a^{\prime}(s) v_{x t}(x, s) g^{\prime \prime}\left(\bar{v}_{x}^{s}(x, s)\right) v_{x}(x, s) v_{0}^{\prime}(x) \mathrm{d} x \mathrm{~d} s
$$

The term $I_{2}$ can be re-written as

$$
\begin{aligned}
I_{2}= & -\frac{1}{2} \int_{0}^{t} \int_{\Omega} \int_{0}^{+\infty} \xi(\tau, s, x) \frac{\partial}{\partial s}\left|\triangle_{h} v_{x}\right|^{2}(x, s) \mathrm{d} \tau \mathrm{d} x \mathrm{~d} s \\
= & -\frac{1}{2} \int_{\Omega} \int_{0}^{+\infty} \xi(\tau, t, x)\left|\triangle_{h} v_{x}(x, t)\right|^{2} \mathrm{~d} \tau \mathrm{d} x \\
& +\frac{1}{2} \int_{0}^{t} \int_{\Omega} \int_{0}^{+\infty} \partial_{2} \xi(\tau, s, x)\left|\triangle_{h} v_{x}(x, s)\right|^{2} \mathrm{~d} \tau \mathrm{d} x \mathrm{~d} s
\end{aligned}
$$

Dividing by $h^{2}$ and passing to the limit for $h \rightarrow 0_{+}$one obtains

$$
\frac{1}{h^{2}} I_{2} \underset{h \rightarrow 0_{+}}{\longrightarrow} J_{2}
$$

where

$$
\begin{aligned}
J_{2}= & -\frac{1}{2} \int_{\Omega} \int_{0}^{+\infty} \xi(\tau, t, x)\left|v_{x t}(x, t)\right|^{2} \mathrm{~d} \tau \mathrm{d} x \\
& +\frac{1}{2} \int_{0}^{t} \int_{\Omega} \int_{0}^{+\infty} \partial_{2} \xi(\tau, s, x)\left|v_{x t}(x, s)\right|^{2} \mathrm{~d} \tau \mathrm{d} x \mathrm{~d} s
\end{aligned}
$$

Next, $I_{3}=I_{31}+I_{32}+I_{33}$, where

$$
\begin{gathered}
I_{31}=g^{\prime}(0) \int_{0}^{t} \int_{\Omega} \int_{0}^{s} a^{\prime}(\tau) \triangle_{h} v_{x t}(x, s) \triangle_{h} v_{x}(x, s-\tau) \mathrm{d} \tau \mathrm{d} x \mathrm{~d} s \\
I_{32}=g^{\prime}(0) \int_{0}^{t} \int_{\Omega} \int_{s}^{s+h} a^{\prime}(\tau) \triangle_{h} v_{x t}(x, s) v_{x}(x, s+h-\tau) \mathrm{d} \tau \mathrm{d} x \mathrm{~d} s \\
I_{33}=g^{\prime}(0) a(0) \int_{0}^{t} \int_{\Omega} \triangle_{h} v_{x t}(x, s) \triangle_{h} v_{x}(x, s) \mathrm{d} x \mathrm{~d} s
\end{gathered}
$$

Upon integration by parts w.r.t. $\tau$ leads to

$$
\begin{aligned}
I_{31} & =g^{\prime}(0) \int_{0}^{t} \int_{\Omega} a(s) \triangle_{h} v_{x t}(x, s) \triangle_{h} v_{x}(x, 0) \mathrm{d} x \mathrm{~d} s \\
& -g^{\prime}(0) a(0) \int_{0}^{t} \int_{\Omega} \triangle_{h} v_{x t}(x, s) \triangle_{h} v_{x}(x, s) \mathrm{d} x \mathrm{~d} s \\
& +g^{\prime}(0) Q\left(\triangle_{h} v_{x t}, a, t\right)
\end{aligned}
$$

The above implies, upon simplification and integration by parts w.r.t. $s$, that

$$
I_{3}=g^{\prime}(0) Q\left(\triangle_{h} v_{x t}, a, t\right)+g^{\prime}(0) \int_{\Omega} a(t) \triangle_{h} v_{x}(t) \triangle_{h} v_{x}(0) \mathrm{d} x
$$




$$
\begin{aligned}
& -g^{\prime}(0) a(0) \int_{\Omega}\left(\triangle_{h} v_{x}(0)\right)^{2} \mathrm{~d} x-g^{\prime}(0) \int_{0}^{t} \int_{\Omega} a^{\prime}(s) \triangle_{h} v_{x}(s) \triangle_{h} v_{x}(0) \mathrm{d} x \mathrm{~d} s \\
& -g^{\prime}(0) \int_{0}^{t} \int_{\Omega} \int_{s}^{s+h} a^{\prime}(\tau) \triangle_{h} v_{t}(x, s) v_{x x}(x, s+h-\tau) \mathrm{d} \tau \mathrm{d} x \mathrm{~d} s
\end{aligned}
$$

Divide the above by $h^{2}$ and taking the lower limit for $h \rightarrow 0_{+}$gives

$$
\liminf _{h \rightarrow 0_{+}} \frac{1}{h^{2}} I_{3}=g^{\prime}(0) \liminf _{h \rightarrow 0_{+}} \frac{1}{h^{2}} Q\left(\triangle_{h} v_{x t}, a, t\right)+J_{3}
$$

where

$$
\begin{aligned}
J_{3} & =g^{\prime}(0)\left\{a(t) \int_{\Omega} v_{x t}(x, t) v_{x t}(x, 0) \mathrm{d} x-a(0) \int_{\Omega} v_{x t}^{2}(x, 0) \mathrm{d} x\right. \\
& \left.-\int_{0}^{t} \int_{\Omega} a^{\prime}(s) v_{x t}(x, s) v_{x t}(x, 0) \mathrm{d} x \mathrm{~d} s-\int_{0}^{t} \int_{\Omega} a^{\prime}(s) v_{t t}(x, s) v_{0}^{\prime \prime}(x) \mathrm{d} x \mathrm{~d} s\right\}
\end{aligned}
$$

Next we end up with the same result as in 7.37 with $\left(\liminf _{h \rightarrow 0_{+}}\right)$being replaced by $\left(\limsup _{h \rightarrow 0_{+}}\right)$. Now we can write $I_{4}$ in the form:

$$
I_{4}=\int_{0}^{t} \int_{\Omega}\left[\int_{0}^{s} \xi(\tau, s) \triangle_{h} v_{x}(s-\tau) \mathrm{d} \tau+\int_{s}^{s+h} \xi(\tau, s) v_{x}(s+h-\tau) \mathrm{d} \tau\right] \triangle_{h} v_{x t}(x, s) \mathrm{d} x \mathrm{~d} s
$$

An integration by parts w.r.t. $s$ gives

$$
I_{4}=I_{41}+I_{42}+I_{43}+I_{44}
$$

where

$$
\begin{aligned}
I_{41} & =-\int_{0}^{t} \int_{\Omega} \int_{0}^{s}\left[\partial_{2} \xi(\tau, s) \triangle_{h} v_{x}(x, s-\tau)+\xi(\tau, s) \triangle_{h} v_{x t}(x, s-\tau)\right] \mathrm{d} \tau \triangle_{h} v_{x}(x, s) \mathrm{d} x \mathrm{~d} s \\
I_{42} & =-\int_{0}^{t} \int_{\Omega} \int_{s}^{s+h}\left[\partial_{2} \xi(\tau, s) v_{x}(x, s+h-\tau)+\xi(\tau, s) v_{x t}(x, s+h-\tau)\right] \mathrm{d} \tau \triangle_{h} v_{x}(x, s) \mathrm{d} x \mathrm{~d} s
\end{aligned}
$$

$$
I_{43}=-\int_{0}^{t} \int_{\Omega}[\xi(s+h, s)-\xi(s, s)] v_{0}^{\prime}(x) \triangle_{h} v_{x}(x, s) \mathrm{d} x \mathrm{~d} s
$$

and

$$
I_{44}=\left[\int_{\Omega} \int_{0}^{s+h} \xi(\tau, s) \triangle_{h} v_{x}(x, s-\tau) \triangle_{h} v_{x}(x, s) \mathrm{d} \tau \mathrm{d} x\right]_{s=0}^{s=t}
$$

We now deal with the second term in $I_{41}$; we have:

$$
-\int_{0}^{s} \xi(\tau, s) \triangle_{h} v_{x t}(x, s-\tau) \mathrm{d} \tau=\xi(s, s)\left[v_{x}(h)-v_{x}(0)\right]-\int_{0}^{s} \partial_{1} \xi(\tau, s) \triangle_{h} v_{x}(x, s-\tau) \mathrm{d} \tau
$$


fact that allows to get

$$
\begin{aligned}
I_{41} & =-\int_{0}^{t} \int_{\Omega} \int_{0}^{s}\left[\partial_{1} \xi(\tau, s)+\partial_{2} \xi(\tau, s)\right] \triangle_{h} v_{x}(x, s-\tau) \triangle_{h} v_{x}(x, s) \mathrm{d} \tau \mathrm{d} x \mathrm{~d} s \\
& +\int_{0}^{t} \int_{\Omega} \xi(s, s)\left[v_{x}(h)-v_{x}(0)\right] \triangle_{h} v_{x}(x, s) \mathrm{d} x \mathrm{~d} s
\end{aligned}
$$

Now we obtain

$$
\frac{1}{h^{2}} I_{4} \underset{h \rightarrow 0_{+}}{\longrightarrow} J_{4}+J_{04}
$$

where

$$
\begin{aligned}
J_{4}= & -\int_{0}^{t} \int_{\Omega} \int_{0}^{s}\left[\partial_{1} \xi(\tau, s)+\partial_{2} \xi(\tau, s)\right] v_{x t}(x, s-\tau) v_{x t}(x, s) \mathrm{d} \tau \mathrm{d} x \mathrm{~d} s \\
& +\int_{0}^{t} \int_{\Omega} \xi(\tau, t) v_{x t}(x, t-\tau) v_{x t}(x, t) \mathrm{d} x \mathrm{~d} \tau
\end{aligned}
$$

and

$$
J_{04}=-\int_{0}^{t} \int_{\Omega}\left[\partial_{1} \xi(s, s)+\partial_{2} \xi(s, s)\right] v_{0}^{\prime}(x) v_{x t}(x, s) \mathrm{d} x \mathrm{~d} s
$$

Now, from (7.16), (7.17), 7.26), (7.27), (7.30), 7.31), (7.37), 7.38), 7.47), (7.48), we deduce that

$$
\begin{aligned}
& \frac{1}{2} \int_{\Omega} v_{t t}^{2}(x, t) \mathrm{d} x-\frac{1}{2} \int_{\Omega} v_{t t}^{2}(x, 0) \mathrm{d} x=g^{\prime}(0) \lim _{h \rightarrow 0_{+}} \frac{1}{h^{2}} Q\left(\triangle_{h} v_{x t}, a, t\right) \\
& +\int_{0}^{t} \int_{\Omega} v_{t t}(x, s) f_{t t}(x, s) \mathrm{d} x \mathrm{~d} s+J_{1}+J_{2}+J_{3}+J_{4}+J_{01}+J_{04}
\end{aligned}
$$

with $J_{1^{-}} J_{4}$ being given by $(7.27),(7.31),(7.38)$ and $(7.48)$, respectively. One now needs to appropriately bound the terms $J_{1^{-}} J_{4}, J_{01}$ and $J_{04}$. It may be easily seen, using Lemma 7.1. that all terms $J_{1}, J_{2}$ and $J_{4}$ can be bounded by one of the following type of expressions:

$$
c \nu^{k}(t) \int_{\Omega}\left|w_{1}(x, t)\right|\left|w_{2}(x, t)\right| \mathrm{d} x
$$

or

$$
c \nu^{k}(t) \int_{0}^{t} \int_{\Omega}\left|w_{1}(x, s)\right|\left|w_{2}(x, s)\right| \mathrm{d} x \mathrm{~d} s
$$

or

$$
c \nu^{k}(t) \int_{0}^{t} \int_{\Omega} \varphi(\tau)\left|w_{1}(x, t-\tau)\right|\left|w_{2}(x, t)\right| \mathrm{d} x \mathrm{~d} \tau
$$

or

$$
c \nu^{k}(t) \int_{0}^{t} \int_{\Omega} \int_{0}^{s} \varphi(\tau)\left|w_{1}(x, s-\tau)\right|\left|w_{2}(x, s)\right| \mathrm{d} \tau \mathrm{d} x \mathrm{~d} s
$$


where $\varphi \geq 0$ is a given function in $L^{1}\left(\mathbb{R}_{+}\right)$depending on $a, c>0$ is a constant, $w_{1}, w_{2}$ stand for either $v$ or one of its derivatives up to second order, and $k \in\{1,2,3\}$. This is a consequence of assumption $\left(a_{3}\right)$.

Terms like (7.51) and (7.52) can easily be bounded by $c \nu^{k}(t) \mathcal{E}(t)$. Using Lemma 4.2 , terms like (7.53) and (7.54) can also be easily bounded by $c \nu^{k}(t) \mathcal{E}(t)$. We then obtain that there exists a constant $c>0$ s.t.

$$
J_{1}+J_{2}+J_{4} \leq c\left[\nu(t)+\nu^{3}(t)\right] \mathcal{E}(t)
$$

The estimates for $J_{3}, J_{01}$ and $J_{04}$ are simpler to obtain since they contain initial data. Using (4.33) we get $v_{x t}(x, 0)=f_{x}(x, 0)$. It easily follows that

$$
\begin{gathered}
\left|J_{3}\right| \leq\left|g^{\prime}(0)\right|\left(|a(t)|+\left\|a^{\prime}\right\|_{L^{1}\left(\mathbb{R}_{+}\right)}\right)\left[\left(\sqrt{F}+\sqrt{V_{0}}\right) \sqrt{\mathcal{E}(t)}+a(0) F\right] \\
\left|J_{01}\right| \leq K \theta\left\|a^{\prime}\right\|_{L^{1}\left(\mathbb{R}_{+}\right)}\left\|v_{0}\right\|_{H^{2}(\Omega)} \mathcal{E}(t)
\end{gathered}
$$

and

$$
\left|J_{04}\right| \leq 3 K\left(\theta\left\|a^{\prime}\right\|_{L^{1}\left(\mathbb{R}_{+}\right)}+\left\|a^{\prime \prime} r_{0}\right\|_{L^{1}\left(\mathbb{R}_{+}\right)}\right)\left\|v_{0}\right\|_{H^{2}(\Omega)} \nu(t) \sqrt{\mathcal{E}(t)}
$$

From (7.50), 7.55), 7.56) and (7.58), the result stated in the Lemma now follows.

\section{References}

[1] Aarts A.C.T. and van de Ven A.A.F.: Transient behaviour and stability points of the Poiseuille flow of a KBKZ-fluid. Journal of Engineering Mathematics 29(4), 371-392 (1995).

[2] Bae H. and Trivisa K.: On the Doi model for the suspensions of rod-like molecules: globalin-time existence. Communications in Mathematical Sciences 11(3), 831-850 (2013).

[3] Barrett J. W., Schwab C. and Süli E.: Existence of global weak solutions for some polymeric flow models. Mathematical Models \& Methods in Applied Sciences 15(6), 12111289 (2011).

[4] Barrett J. W and Süli E.: Existence and equilibration of global weak solutions to kinetic models for dilute polymers I: finitely extensible nonlinear bead-spring chains. Mathematical Models \& Methods in Applied Sciences 21(6), 1211-1289 (2005).

[5] Barrett J. W and Süli E.: Existence and equilibration of global weak solutions to kinetic models for dilute polymers II: Hookean-type models. Mathematical Models \& Methods in Applied Sciences 22(5), 1150024 (2006).

[6] Bird R. B., Armstrong R. C. and Hassager O.: Dynamics of Polymeric Liquids, Vol. 2: Kinetic Theories, J. Wiley \& Sons, New-York (1987).

[7] Blanc X., Le Bris C. and Lions P. -L.: From molecular models to continuum mechanics. Archives for Rational Mechanics and Analysis 164(4), 341-381 (2002).

[8] Brandon D. and Hrusa W. J.: Global existence of smooth shearing motions of a nonlinear viscoelastic fluid. Journal of Integral Equations and Applications 2(3), 333-351 (1990). 
[9] Brandon D.: Global existence and asymptotic stability for a nonlinear integro-differential equation modeling heat flow. SIAM Journal of Mathematical Analysis 22(1), 72-106 (1991).

[10] Busuioc A. V., Ciuperca I. S., Iftimie D. and Palade L. I.: The FENE dumbbell polymer model: existence and uniqueness of solutions for the momentum balance equation. Journal of Dynamics and Differential Equations 26(2), 217-241 (2014).

[11] Chupin, L.: Fokker-Planck equation in bounded domain. Annales de l'Institut Fourier 60(1), 217-255 (2010).

[12] Ciuperca I. S., Heibig A.: Existence and uniqueness of a density probability solution for the stationary Doi-Edwards equation, Annales de l'Institut Henri Poincaré - Analyse non linéaire, doi:10.1016/j.anihpc.2015.05.003, accepted for publication, 2015.

[13] Ciuperca I. S., Heibig A. and Palade L. I.: Existence and uniqueness results for the DoiEdwards polymer melt model: the case of the (full) nonlinear configurational probability density equation. Nonlinearity 25(4), 991-1009 (2012).

[14] Ciuperca I. S. and Palade L. I.: The steady state configurational distribution diffusion equation of the standard FENE dumbbell polymer model: existence and uniqueness of solutions for arbitrary velocity gradients. Mathematical Models \& Methods in Applied Sciences 19, 2039-2064 (2009).

[15] Ciuperca I. S. and Palade L. I.: On the existence and uniqueness of solutions of the configurational probability diffusion equation for the generalized rigid dumbbell polymer model. Dynamics of Partial Differential Equations 7, 245-263 (2010).

[16] Ciuperca I. S. and Palade L. I.: Asymptotic behavior of the solution of the distribution diffusion equation for FENE dumbbell polymer model. Mathematical Modelling of Natural Phenomena 6(5), 84-97 (2011).

[17] Constantin P. and Masmoudi N.: Global well-posedness for a Smoluchowski equation coupled with Navier-Stokes equations in 2D. Communications in Mathematical Physics 278, 179-191 (2008).

[18] de Gennes P. -G.: Scaling Concepts in Polymer Physics. Cornell University Press, Ithaca NJ and London (1979).

[19] Doi M. and Edwards S. F.: Dynamics of concentrated polymer systems, Part 3.-The constitutive equation. Journal of the Chemical Society, Faraday Transactions II 74, 18181832 (1978).

[20] Doi M. and Edwards S. F.: The Theory of Polymer Dynamics. Oxford University Press, Oxford (1989).

[21] Degond P., Lemou M. and Picasso M.: Viscoelastic fluid models derived from kinetic equations for polymers. SIAM Journal of Applied Mathematics 62(5), 1501-1519 (2002).

[22] Engler H.: Weak solutions of a class of quasilinear hyperbolic integro-differential equations describing viscoelastic materials. Archives for Rational Mechanics and Analysis 113(1), 1-38, 1991; ibid 130(4), 401 (1995).

[23] Eller M. M.: Gårding's inequality on manifolds with boundary, Chap. 6, 87-99, in Control Theory of Partial Differential Equations, O. Imanuvilev, G. Leugering, R. Triggiani and B. Zhang Editors, Chapman \& Hall/CRC, Boca Raton FL (2005). 
[24] Gripenberg G., Londen S. -O. and Staffans O.: Volterra Integral and Functional Equations. Cambridge University Press (1990).

[25] Hrusa W. J. and Renardy M. A model equation for viscoelasticity with a strongly singular kernel. SIAM Journal of Mathematical Analysis, 19, 257-269 (1988).

[26] Jourdain B., Le Bris C., Lelievre T. and Otto F.: Long-time asymptotics of a multiscale model for polymeric fluid flows. Archives for Rational Mechanics and Analysis 181(1), 97-148 (2006).

[27] Kim J. U. Global smooth solutions of the equations of motion of a nonlinear fluid with fading memory. Archive for Rational Mechanics and Analysis, 79, 97-130 (1982).

[28] Le Bris C. and Lions P. -L.: Existence and uniqueness of solutions to Fokker-Planck type equations with irregular coefficients. Communications in Partial Differential Equations 33(7), 1272-1317 (2008).

[29] Lions J. -L and Magenes E.: Problèmes aux Limites Non-homogènes et Applications. vol. 1, Dunod, Paris (1968).

[30] Masmoudi N.: Well-posedness for the FENE dumbbell model of polymeric flows. Communications in Pure and Applied Mathematics 61(12), 1685-1714, (2008).

[31] Masmoudi N.: Global existence of weak solutions to the FENE dumbbell model of polymeric flows. Inventiones Mathematicae 191, 427-500 (2013).

[32] Öttinger H. -C.: Beyond Equilibrium Thermodynamics. Wiley (2006).

[33] Otto F. and Tzavaras A. E.: Continuity of velocity gradients in suspensions of rod-like molecules. Communications in Mathematical Physics 277(3), 729-758 (2008).

[34] Palade L. I.: On slow flows of the full nonlinear Doi-Edwards polymer model. Zeitschrift für Angewandte Mathematik und Physik ZAMP 65, 139-148 (2014).

[35] Palierne J. F.: Rheothermodynamics of the Doi-Edwards reptation model, Phys. Rev. Lett. 93: 136001-1 - 136001-4, 2004.

[36] Prüss J.: Evolutionary Integral Equations and Applications. Modern Birkhaüser Classics, Springer, New York NY (2012).

[37] Renardy M., Hrusa W. J. and Nohel J. A.: Mathematical Problems in Viscoelasticity. Pitman Monographs and Surveys in Pure and Applied Mathematics vol. 35, Longman Scientific \& Technical, Harlow Essex (1987).

[38] Renardy M.: Mathematical Analysis of Viscoelastic Flows, SIAM (2000).

[39] Staffans O. J.: On a nonlinear hyperbolic Volterra equation. SIAM Journal of Mathematical Analysis 11, 793-812 (1980).

[40] Stein E. M.: Singular Integrals and Differentiability Properties of Functions, Princeton University Press, Princeton NJ (1970).

[41] Taylor M. E.: Pseudodifferential operators. Princeton Mathematical Series, 34. Princeton University Press, Princeton NJ (1981).

[42] Zhang H. and Zhang P.: Local existence for the FENE-dumbbell model of polymeric fluids. Archives for Rational Mechanics and Analysis 181(2), 373-400 (2006). 